Research Article

\title{
Forgotten Index of Generalized Operations on Graphs
}

\author{
Muhammad Javaid $(1),{ }^{1}$ Saira Javed $\left(\mathbb{D},{ }^{1}\right.$ Saima Q. Memon, ${ }^{2}$ \\ and Abdulaziz Mohammed Alanazi $\mathbb{D D}^{3}$ \\ ${ }^{1}$ Department of Mathematics, School of Science, University of Management and Technology, Lahore 54770, Pakistan \\ ${ }^{2}$ M. A. Kazi Institute of Chemistry, University of Sindh, Jamshoro, Pakistan \\ ${ }^{3}$ Department of Mathematics, University of Tabuk, Tabuk 71491, Saudi Arabia
}

Correspondence should be addressed to Muhammad Javaid; javaidmath@gmail.com

Received 4 March 2021; Revised 21 March 2021; Accepted 27 March 2021; Published 3 May 2021

Academic Editor: Kashif Ali

Copyright (c) 2021 Muhammad Javaid et al. This is an open access article distributed under the Creative Commons Attribution License, which permits unrestricted use, distribution, and reproduction in any medium, provided the original work is properly cited.

\begin{abstract}
In theoretical chemistry, several distance-based, degree-based, and counting polynomial-related topological indices (TIs) are used to investigate the different chemical and structural properties of the molecular graphs. Furtula and Gutman redefined the $F$-index as the sum of cubes of degrees of the vertices of the molecular graphs to study the different properties of their structuredependency. In this paper, we compute $F$-index of generalized sum graphs in terms of various TIs of their factor graphs, where generalized sum graphs are obtained by using four generalized subdivision-related operations and the strong product of graphs. We have analyzed our results through the numerical tables and the graphical presentations for the particular generalized sum graphs constructed with the help of path (alkane) graphs.
\end{abstract}

\section{Introduction}

Throughout the paper, we consider a simple and undirected graph $H=(V(H), E(H))$ with vertex-set $V(H)=\left\{v_{1}\right.$, $\left.v_{2}, v_{3}, \ldots, v_{n}\right\}$ and edge-set $E=\left\{e_{1}, e_{2}, \ldots, e_{m}\right\} \subseteq$ $V(H) \times V(H)$, where $n=|V(G)|$ and $m=|E(G)|$. A molecular graph is a connected and undirected graph in which atoms are presented by vertices, and chemical bonds between these atoms are shown by edges (see Figure 1). For a finite set of graphs $\mathscr{C}$ and set of real numbers $\mathbb{R}$, the function $I: \mathscr{C} \longrightarrow \mathbb{R}$ defined by $I(H)=\sum_{u \in V(H)} f(u)$ is called a degree-based topological index (TI), where the graph $H$ belongs to $\mathscr{C}$ and $f(u)$ is a degree-function from the vertex-set of $H$ to the degree-set of its vertices. It is important to know that $I(H)=I(K)$ if and only if $H$ is isomorphic to $K$. For more details, see $[1,2]$.

Graph-theoretic modeling of the molecular graphs plays a fundamental part in the analysis of the quantitative structures activity/property relationships (QSAR/QSPR). In chemistry, the study of structural relationships is used to characterize the various physicochemical properties of organic molecules such as surface tension, density, melting, freezing point, solubility, heat of evaporation, and heat of formation [3]. In last two decades, many TIs are introduced, but degree-based TIs got much more attention of the researchers, see the latest survey [4]. In 1947, Winer introduced the first distance-based TI to compute the boiling point of paraffin [5]. Also, we refer [6].

In molecular graph theory, the different operations on a graph perform a fundamental role in the formation of different new classes of graphs. Yan et al. [7] introduced the four operations $S_{1}, R_{1}, Q_{1}$, and $T_{1}$ on a graph $H$ and computed the Wiener indices for the graphs $F_{1}(H)$, where $F_{1} \in\left\{S_{1}, R_{1}, Q_{1}, T_{1}\right\}$. Eliasi and Taeri [8] defined the $F_{1}$-sum graphs $\left(H_{1+F_{1}} H_{2}\right)$ using the Cartesian product on graphs $F_{1}\left(H_{1}\right)$ and $H_{2}$, where $H_{1}$ and $H_{2}$ are any two simple and connected graphs. They also computed the Wiener indices for these $F_{1}$-sum graphs. Furthermore, Deng et al. [9] calculated the $M_{1}$ and $M_{2}$ Zagreb indices, Imran and Shehnaz [10] computed the F-index, Liu et al. [11] computed the first general Zagreb, Chu et al. [12] calculated the bounds of first general Zagreb index and general Randic index, and 


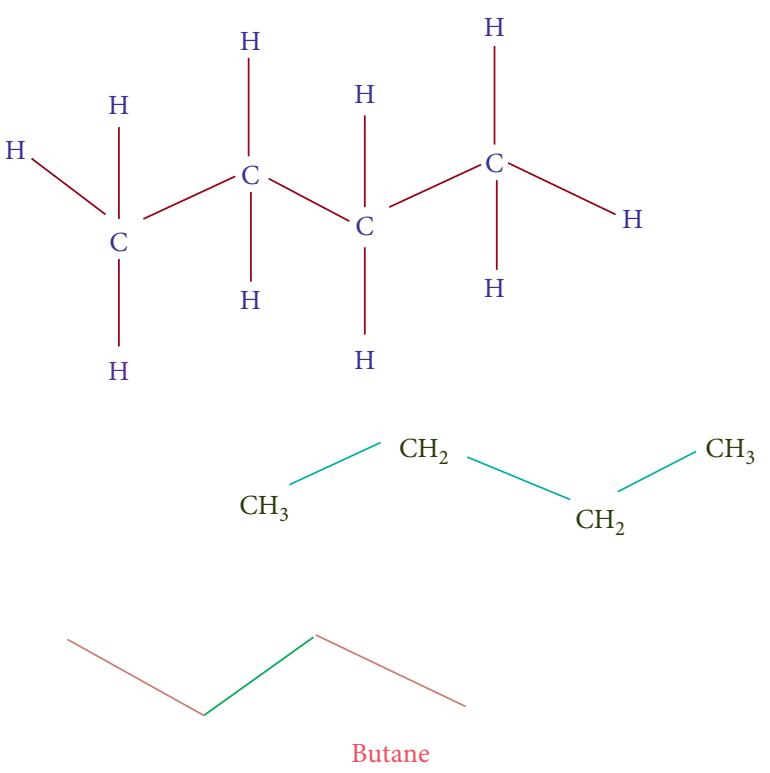

Figure 1: Clearly, structural formula of butane is isomorphic to $P_{4}$.

Sarala et al. [13] computed the $F$-index for these $F_{1}$-sum graphs under the Cartesian and strong products. We also refer [14-20].

Recently, for $k \geq 1$, Liu et al. [21] defined the generalized $F$-sum $\left(F_{k}\right.$-sum) graphs using Cartesian product and computed their Zagreb indices. Moreover, Awais et al. computed the F-index [22], hyper-Zagreb [23], and FGZ index [24] for these generalized $F$-sum graphs. Recently, Javaid et al. [25] computed first and second Zagreb indices for the generalized $F$-sum graphs under the strong product.

In this paper, we compute $F$-index of generalized sum graphs in terms of various TIs of their factor graphs, where generalized sum graphs are obtained by using four generalized subdivision-related operations and the strong product of graphs. We have analyzed our results through the numerical tables and the graphical presentations for the particular generalized sum graphs constructed with the help of path (alkane) graphs. The remaining paper is managed as follows: Section 2 consists of elementary definitions, Section 3 includes main results, and Section 4 covers the application and conclusion.

\section{Preliminaries}

2.1. Degree-Based Topological Indices. In 1972, Trainajsi and Gutman defined first and second Zagreb indices that are utilized to find the $\pi$-electron energy of molecular graphs [26]. Let $H$ be any graph (molecular structure), then the first and second Zagreb indices are defined as $M_{1}(H)=\sum_{v \in V(H)} d^{2}(v)$ and $M_{2}(H)=\sum_{u v \in E(H)} d(u) d(v)$.

In 2015, Furtula and Gutman [22] redefined a TI called by forgotten TI [27]. The forgotten TI of a (molecular) graph $H$ is defined as

$$
F(H)=\sum_{v \in V(H)} d^{3}(v)
$$

They also verified that the different predictive abilities of $F$-index and first Zagreb index are same. In particular, both the indices yield the values of correlation coefficients for entropy and acentric factor more than 0.95 . For more results on its mathematical properties and chemical applications, see [23].

2.2. Four Generalized Operations. Let $H_{1}$ be a (molecular) graph, then for the integral value $k \geq 1$, the graphs $\left(F_{k}\left(H_{1}\right)\right.$ under the four generalized operations $\left(F_{k} \in\left\{S_{k}, R_{k}, Q_{k}, T_{k}\right\}\right)$ on $H_{1}$ are defined as follows [21]:

(i) $k$-subdivision graph: we add $k$ new vertices in every edge of $H_{1}$ and obtain the new $k$-subdivision graph $S_{k}\left(H_{1}\right)$

(ii) $k$-semitotal point graph: the graph $k$-semitotal point $\left(R_{k}\left(H_{1}\right)\right)$ is defined from the graph $S_{k}\left(H_{1}\right)$ by joining the vertices of $S_{k}\left(H_{1}\right)$ which were adjacent in $H_{1}$

(iii) $k$-semitotal line graph: the $k$-semitotal line graph $Q_{k}\left(H_{1}\right)$ is defined from the graph $S_{k}\left(H_{1}\right)$ by joining the newly added $k$ vertices for each incident pair of edges of $H_{1}$

(iv) $k$-total point graph: the $k$-total point graph is defined from the graph $S_{k}\left(H_{1}\right)$ by applying both the operations $R_{k}$ and $Q_{k}$, respectively. For more details, see Figures 2-4.

2.3. Generalized Sum Operation for Strong Product. Let $H_{1}$ and $H_{2}$ be two graphs, $F_{k} \in\left\{S_{k}, R_{k}, Q_{k}, T_{k}\right\}$ presents generalized operations and $F_{k}\left(H_{1}\right)$ is obtained after applying $F_{k}$ on $H_{1}$ having edge-set $E\left(F_{k}\left(H_{1}\right)\right)$ and node-set $V\left(F_{k}\left(H_{1}\right)\right)$. The generalized $F$-sum graphs $\left(H_{1 F_{k}} \otimes H_{2}\right)$ under the operation of strong product is a graph having a vertex-set: 


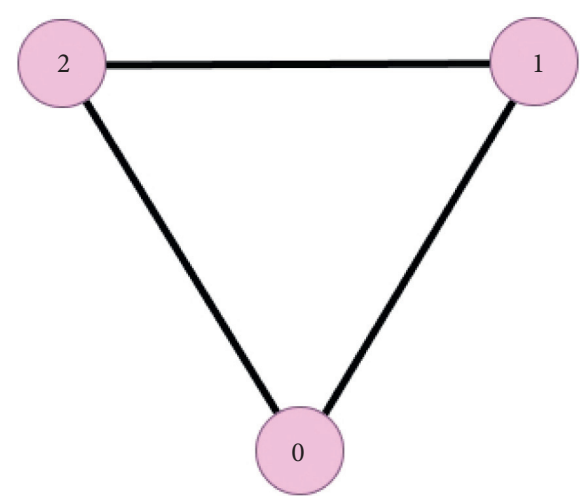

(a)

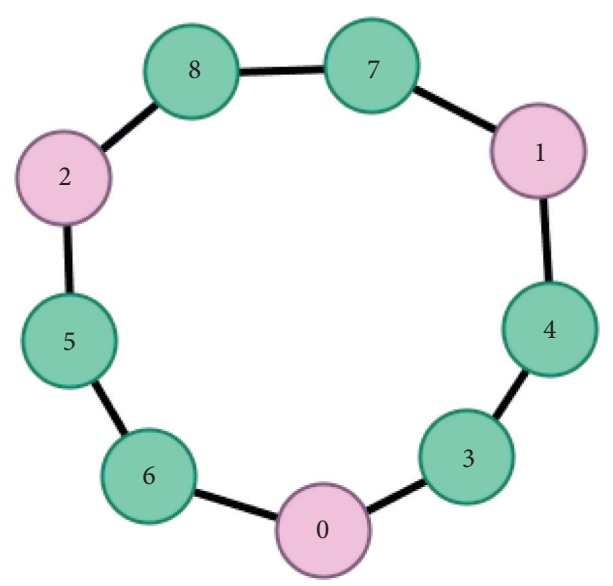

(b)

Figure 2: (a) $H_{1} \cong C_{3}$. (b) $S_{3}\left(H_{1}\right)$.

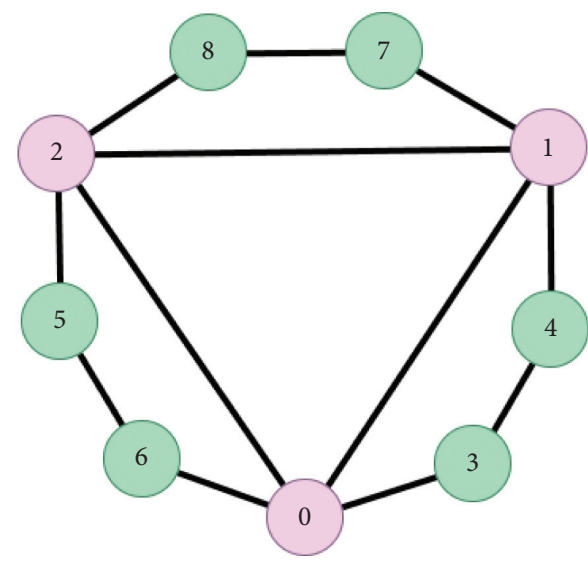

(a)

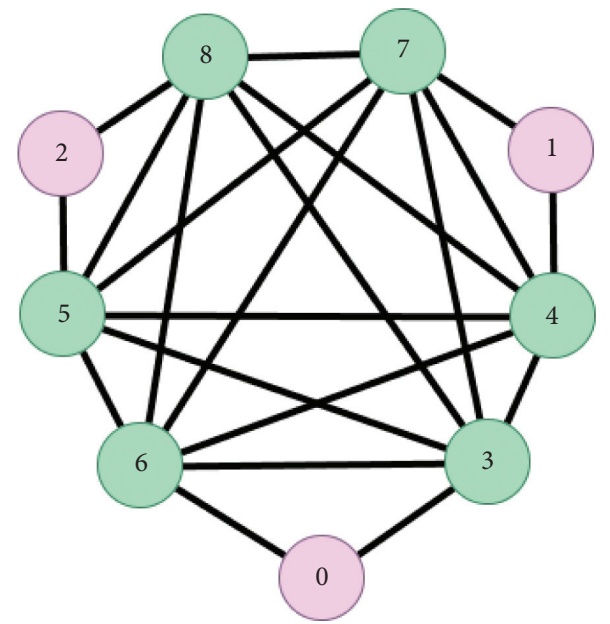

(b)

Figure 3: (a) $R_{3}\left(H_{1}\right)$. (b) $Q_{3}\left(H_{1}\right)$.

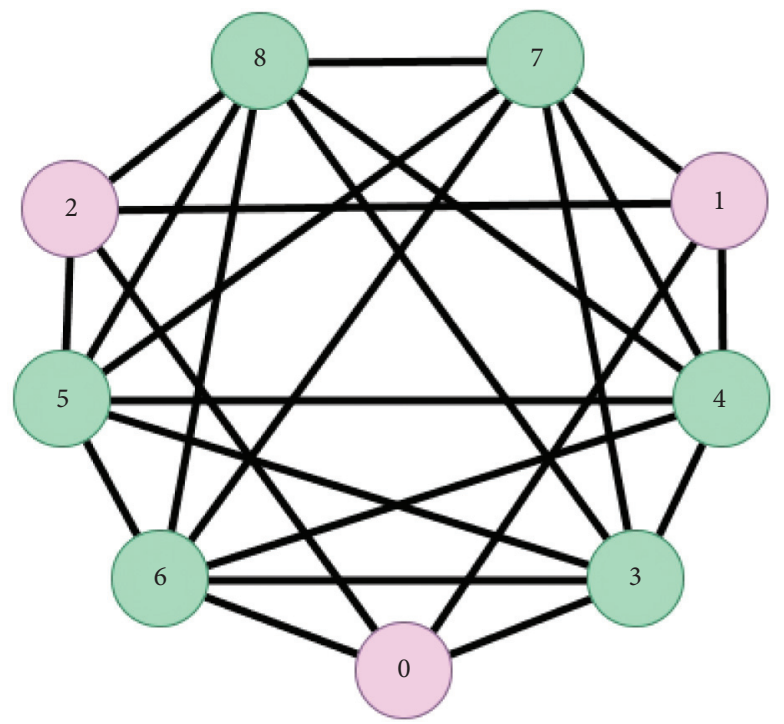

Figure 4: $T_{3}\left(H_{1}\right)$. 


$$
\begin{aligned}
V\left(H_{1 F_{k}} \otimes H_{2}\right) & =V\left(F_{k}\left(H_{1}\right)\right) \times V\left(H_{2}\right) \\
& =\left(V\left(H_{1}\right) \cup k E\left(H_{1}\right)\right) \times V\left(H_{2}\right),
\end{aligned}
$$

such that two vertices $\left(s_{1}, t_{1}\right)$ and $\left(s_{2}, t_{2}\right)$ of $V\left(H_{1 F_{k}} \otimes H_{2}\right)$ are adjacent iff $s_{1}=s_{2} \in V\left(H_{1}\right)$ and $\left(t_{1}, t_{2}\right) \in E\left(H_{2}\right)$ or $t_{1}=$ $t_{2} \in V\left(H_{2}\right)$ and $\left(s_{1}, s_{2}\right) \in E\left(F_{k}\left(H_{1}\right)\right)$ or $\left(t_{1}, t_{2}\right) \in E\left(H_{2}\right)$ and $\left(s_{1}, s_{2}\right) \in E\left(F_{k}\left(H_{1}\right)\right)$, where $k \geq 1$ is natural number. Furthermore, the generalized $F$-sum graphs $\left(H_{1 F_{k}} \otimes H_{2}\right)$ contain $\left|V\left(H_{2}\right)\right|$ copies of graphs $F_{k}\left(H_{1}\right)$ that are labeled with the vertices of $\mathrm{H}_{2}$. For more explanation, see Figures 5-7.

\section{Methodology}

This section presents the main results.

Theorem 1. Let $H_{1}$ and $H_{2}$ be two connected graphs, then

$$
\begin{aligned}
F\left(H_{1_{s_{k}}} \otimes H_{2}\right)= & {\left[\left|V\left(H_{2}\right)\right|+3 M_{1}\left(H_{2}\right)+6\left|E\left(H_{2}\right)\right|\right] F\left(H_{1}\right)+\left[\left|V\left(H_{1}\right)\right|+3 M_{1}\left(H_{1}\right)+14\left|E\left(H_{1}\right)\right|\right] F\left(H_{2}\right) } \\
& +6\left|E\left(H_{2}\right)\right| M_{1}\left(H_{1}\right)+30\left|E\left(H_{1}\right)\right| M_{1}\left(H_{2}\right)+6 M_{1}\left(H_{1}\right) M_{1}\left(H_{2}\right)+48 \mid E\left(H_{1}\right)\left\|E\left(H_{2}\right)\right\| \\
& +8(k-1)\left|E\left(H_{1}\right)\right|\left[6\left|E\left(H_{2}\right)\right|+\left|V\left(H_{2}\right)\right|+F\left(H_{2}\right)+3 M_{1}\left(H_{2}\right)\right]+F\left(H_{1}\right) F\left(H_{2}\right) \\
& +8\left|V\left(H_{2}\right)\right| \mid E\left(H_{1}\right)
\end{aligned}
$$

Proof. Let $d(s, t)=d_{H_{1 S_{k}} \otimes H_{2}}(s, t)$ is the degree of the vertex $(s, t)$ in $H_{1_{s_{k}}} \otimes H_{2}$, then ${ }^{k}$

$$
\begin{aligned}
& F\left(H_{s_{s_{k}}} \otimes H_{2}\right)=\sum_{(s, t) \in V\left(H_{1_{S_{k}}} \otimes H_{2}\right)} d(s, t)^{3}=\sum_{\left(s_{1}, t_{1}\right)\left(s_{2}, t_{2}\right) \in E\left(H_{1_{S_{k}}} \otimes H_{2}\right)}\left[d\left(s_{1}, t_{1}\right)^{2}+d\left(s_{2}, t_{2}\right)^{2}\right] \\
& =\sum_{s e V\left(H_{1}\right)} \sum_{t_{1} t_{2} \in E\left(H_{2}\right)}\left[d\left(s, t_{1}\right)^{2}+d\left(s, t_{2}\right)^{2}\right]+\sum_{t \in V\left(H_{2}\right)} \sum_{s_{1} s_{2} \in E\left(s_{k}\left(H_{1}\right)\right)}\left[d\left(s_{1}, t\right)^{2}+d\left(s_{2}, t\right)^{2}\right] \\
& +\sum_{t_{1} t_{2} \in E\left(H_{2}\right)} \sum_{s_{1} s_{2} \in E\left(s_{k}\left(H_{1}\right)\right)}\left[d\left(s_{1}, t_{1}\right)^{2}+d\left(s_{2}, t_{2}\right)^{2}\right]=\sum_{A}+\sum_{B}+\sum_{C} . \\
& \sum_{A}=\sum_{s \in V\left(H_{1}\right)} \sum_{t_{1} t_{2} \in E\left(H_{2}\right)}\left[\left(d(s)+d\left(t_{1}\right)+d(s) d\left(t_{1}\right)\right)^{2}+\left(d(s)+d\left(t_{2}\right)+d(s) d\left(t_{2}\right)\right)^{2}\right] \\
& =\sum_{s \in V\left(H_{1}\right)} \sum_{t_{1} t_{2} E\left(H_{2}\right)}\left[2 d(s)^{2}+\left(d\left(t_{1}\right)^{2}+d\left(t_{2}\right)^{2}\right)+d^{2}(s)\left(d^{2}\left(t_{1}\right)+d^{2}\left(t_{2}\right)\right)\right] \\
& \left.+2 d(s)\left(d\left(t_{1}\right)+d\left(t_{2}\right)\right)+2 d^{2}(s)\left(d\left(t_{1}\right)+d\left(t_{2}\right)\right)+2 d(s)\left(d^{2}\left(t_{1}\right)+d^{2}\left(t_{2}\right)\right)\right] \\
& =2\left|E\left(H_{2}\right)\right| M_{1}\left(H_{1}\right)+\left|V\left(H_{1}\right)\right| F\left(H_{2}\right)+4\left|E\left(H_{1}\right)\right| M_{1}\left(H_{2}\right)+M_{1}\left(H_{1}\right) F\left(H_{2}\right) \\
& +2 M_{1}\left(H_{1}\right) M_{1}\left(H_{2}\right)+4\left|E\left(H_{1}\right)\right| F\left(H_{2}\right), \\
& \sum_{B}=\sum_{t \in V\left(H_{2}\right)} \sum_{s_{1} s_{2} \varepsilon E\left(s_{k}\left(H_{1}\right)\right)}\left[d^{2}\left(s_{1}, t\right)+d^{2}\left(s_{2}, t\right)\right]=\sum_{t \varepsilon V\left(H_{2}\right)} \sum_{\substack{s_{1} \varepsilon V\left(H_{1}\right), s_{2} \varepsilon V\left(s_{k}\left(H_{1}\right)-H_{1}\right)}}\left[d^{2}\left(s_{1}, t\right)+d^{2}\left(s_{2}, t\right)\right] \\
& +\sum_{t \varepsilon V\left(H_{2}\right) s_{1}, s_{2} \varepsilon V\left(S_{k}\left(H_{1}\right)-H_{1}\right)}\left[d^{2}\left(s_{1}, t\right)+d^{2}\left(s_{2}, t\right)\right]=\sum_{B_{1}}+\sum_{B_{2}} . \\
& \sum_{B_{1}}=\sum_{t \varepsilon V\left(H_{2}\right)} \sum_{\substack{s_{1} \in s_{2} E E\left(s_{k}\left(H_{1}\right)\right) \\
s_{2} E V\left(s_{k}\left(H_{1}\right)-H_{1}\right)}}\left[\left(d\left(s_{1}\right)+d(t)+d\left(s_{1}\right) d(t)\right)^{2}+\left(d\left(s_{2}\right)+d\left(s_{2}\right) d(t)\right)^{2}\right] \\
& =\sum_{t \varepsilon V\left(H_{2}\right)} \sum_{\substack{s_{1} \varepsilon s_{2} \varepsilon E\left(S_{k}\left(H_{1}\right)\right) s_{1} \varepsilon V\left(H_{1}\right), s_{2} \varepsilon V\left(S_{k}\left(H_{1}\right)-H_{1}\right)}}\left[d\left(s_{1}\right)^{2}+d\left(s_{2}\right)^{2}+d(t)^{2}\left(d\left(s_{1}\right)^{2}+d\left(s_{2}\right)^{2}\right)\right] \\
& \left.+2 d(t)\left(d\left(s_{1}\right)^{2}+d\left(s_{2}\right)^{2}\right)+2 d(t)\left(d\left(s_{1}\right)+2 d^{2}(t)\right)\left(d\left(s_{1}\right)+d^{2}(t)\right)\right] .
\end{aligned}
$$




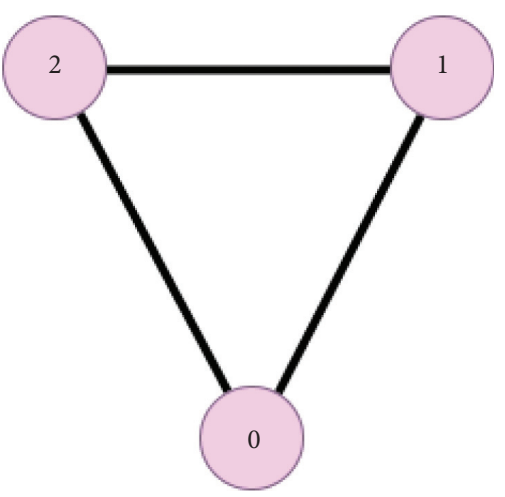

(a)

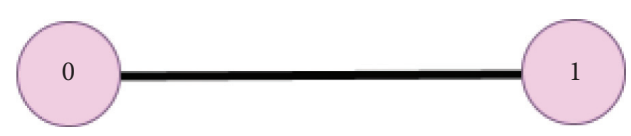

(b)

Figure 5: (a) $H_{1}=C_{3}$. (b) $H_{2}=P_{2}$.

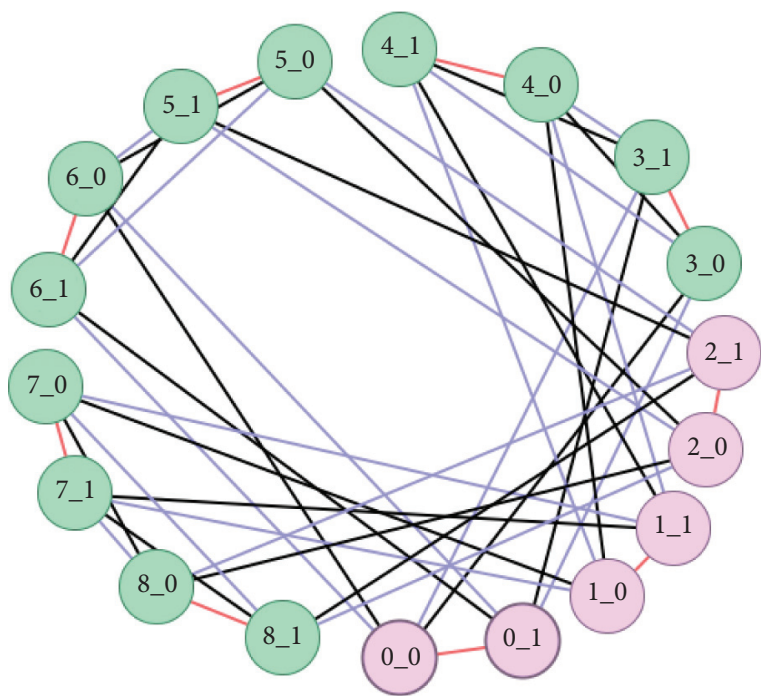

(a)

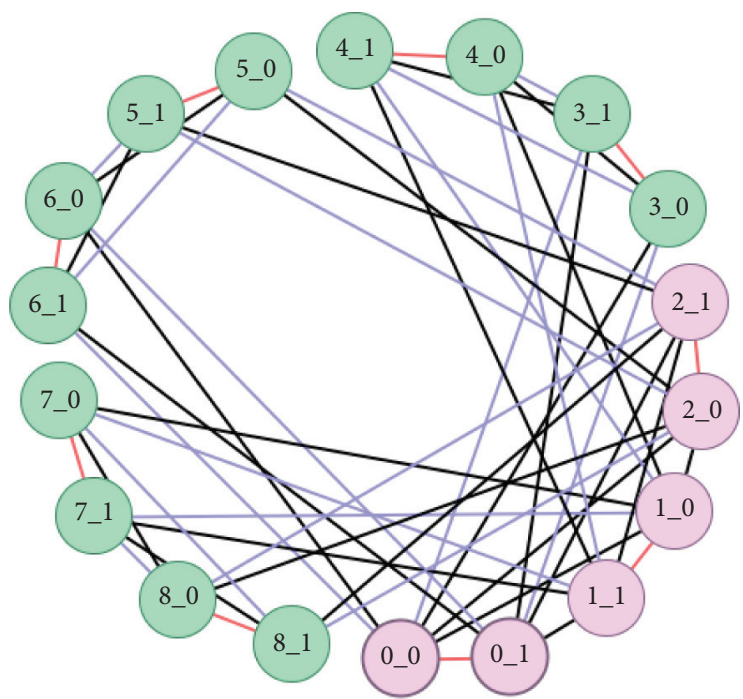

(b)

FIgURE 6: (a) $H_{1_{S_{2}}} \otimes H_{2}$. (b) $H_{1_{R_{2}}} \otimes H_{2}$.

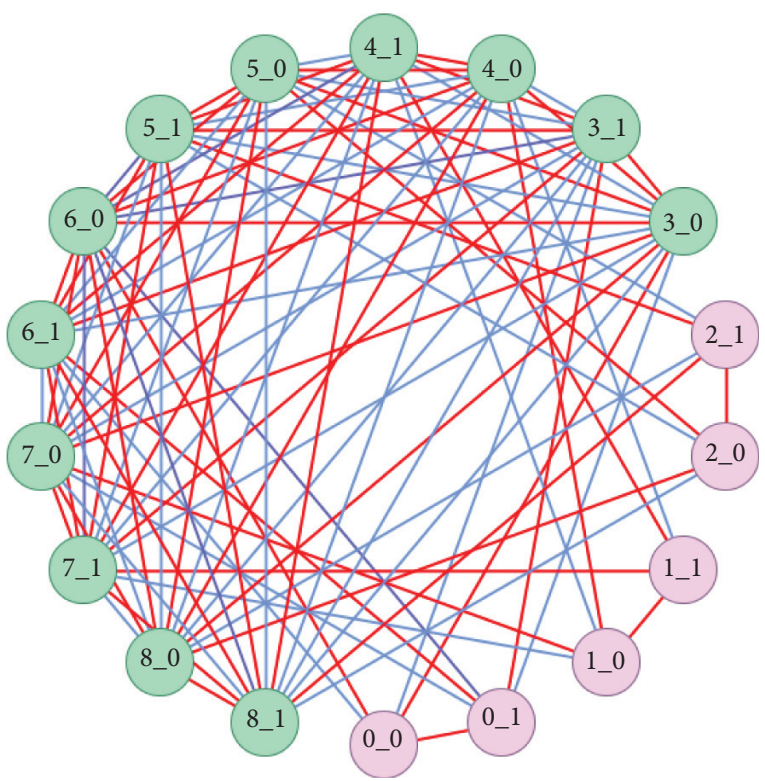

(a)

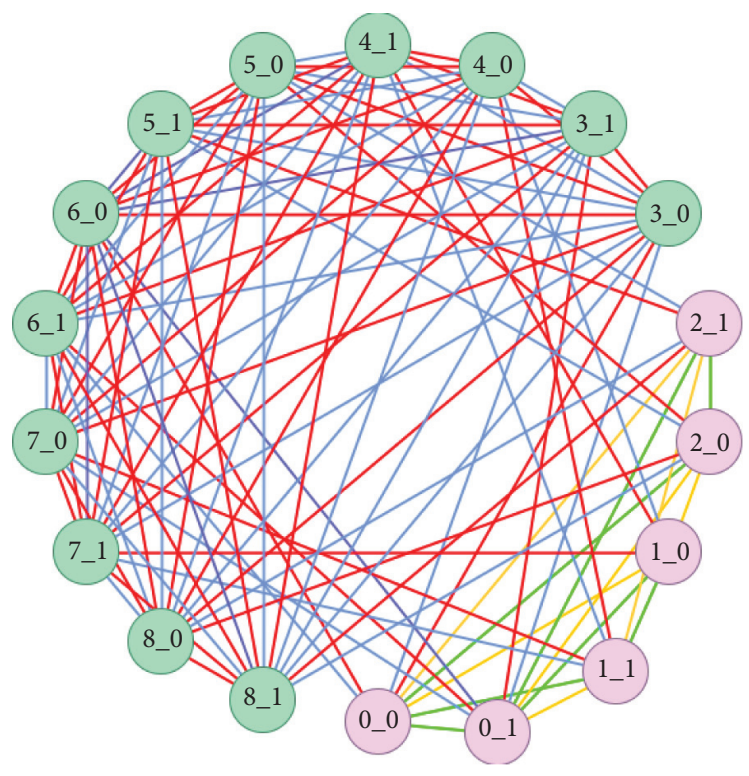

(b)

Figure 7: (a) $H_{1_{Q_{2}}} \otimes H_{2}$. (b) $H_{1_{T_{2}}} \otimes H_{2}$. 
Since $s_{1} \epsilon V\left(H_{1}\right)$ and $\left.s_{2} \epsilon V\left(S_{k}\left(H_{1}\right)-H_{1}\right)\right)$, therefore

$$
\begin{aligned}
= & \left|V\left(H_{2}\right)\right| F\left(S_{1}\left(H_{1}\right)\right)+2\left|E\left(H_{1}\right)\right| M_{1}\left(H_{2}\right)+4\left|E\left(H_{2}\right) M_{1}\left(H_{1}\right)+4\right| E\left(H_{2}\right) F\left(S_{1}\left(H_{1}\right)\right) \\
& +M_{1}\left(H_{2}\right) F\left(S_{1}\left(H_{1}\right)\right)+2 M_{1}\left(H_{1}\right) M_{1}\left(H_{2}\right) .
\end{aligned}
$$

We know that $F\left(S_{1}\left(H_{1}\right)\right)=F\left(H_{1}\right)+8\left|E\left(H_{1}\right)\right|$.

$$
\begin{aligned}
& =\left[\left|V\left(H_{2}\right)\right|+4 \mid E\left(H_{2}\right)+M_{1}\left(H_{2}\right)\right]\left[F\left(H_{1}\right)+8\left|E\left(H_{1}\right)\right|\right]+2\left|E\left(H_{1}\right)\right| M_{1}\left(H_{2}\right)+4 \mid E\left(H_{2}\right) M_{1}\left(H_{1}\right) \\
& +4 \mid E\left(H_{2}\right)+2 M_{1}\left(H_{1}\right) M_{1}\left(H_{2}\right), \\
& \sum_{B_{2}}=\sum_{\substack{t \in V\left(H_{2}\right) \\
s_{1} s_{2} \in E\left(s_{k}\left(H_{1}\right)\right) s_{1}, s_{2} \in V\left(s_{k}\left(H_{1}\right)-H_{1}\right)}}\left[\left(d\left(s_{1}\right)+d\left(s_{1}\right) d(t)\right)^{2}+\left(d\left(s_{2}\right)+d\left(s_{2}\right) d(t)\right)^{2}\right] \\
& =\sum_{t \in V\left(H_{2}\right)} \sum_{\substack{s_{1} s_{2} \in E\left(S_{k}\left(H_{1}\right) s_{1}, s_{2} \in V\left(s_{k}\left(H_{1}\right)-H_{1}\right)\right.}}\left[d\left(s_{1}\right)^{2}+d\left(s_{2}\right)^{2}+d(t)^{2}\left(d\left(s_{1}\right)^{2}+d\left(s_{2}\right)^{2}\right)+2 d(t)\left(d\left(s_{1}\right)^{2}+d\left(s_{2}\right)^{2}\right)\right] \\
& =\sum_{\substack{t \in V\left(H_{2}\right) \\
\sum_{s_{1} s_{2} \in E\left(s_{k}\left(H_{1}\right) s_{1},\right.} \in V\left(s_{k}\left(H_{1}\right)-H_{1}\right)}}\left[(2)^{2}+(2)^{2}+8 d_{H_{2}}(t)^{2}+16 d_{H_{2}}(t)\right] .
\end{aligned}
$$

Since in this case $\left|E\left(S_{k}\left(H_{1}\right)\right)\right|=\left|E\left(H_{1}\right)\right|(k-1)$, so

$$
\begin{aligned}
& =8(k-1)\left|V\left(H_{2}\right)\left\|E\left(H_{1}\right)\right\|\right|+8(k-1) M_{1}\left(H_{2}\right)\left|E\left(H_{1}\right)\right|+32(k-1)\left|E\left(H_{2}\right)\left\|E\left(H_{1}\right)\right\|\right|, \\
& \sum_{C}=\sum_{t_{1} t_{2} \in E\left(H_{2}\right)} \sum_{s_{1} s_{2} \in E\left(S_{k}\left(H_{1}\right)\right)}\left[d^{2}\left(s_{1}, t_{1}\right)+d^{2}\left(s_{2}, t_{2}\right)\right]=\sum_{\substack { t_{1} t_{2} \in E\left(H_{2}\right) \\
\begin{subarray}{c}{s_{1} \in V\left(H_{1}\right), s_{2} \in V\left(s_{k}\left(H_{1}\right)-H_{1}\right){ t _ { 1 } t _ { 2 } \in E ( H _ { 2 } ) \\
\begin{subarray} { c } { s _ { 1 } \in V ( H _ { 1 } ) , \\
s _ { 2 } \in V ( s _ { k } ( H _ { 1 } ) - H _ { 1 } ) } }\end{subarray}}\left[d^{2}\left(s_{1}, t_{1}\right)+d^{2}\left(s_{2}, t_{2}\right)\right] \\
& \left.\left.+\sum_{t_{1} t_{2} \in E\left(H_{2}\right)} \sum_{s_{1}, s_{2} \in V}\left[d^{2}\left(s_{1}, t_{1}\right)+d^{2}\left(s_{1}\right)-H_{1}\right) t_{2}\right)\right]=\sum_{C_{1}}+\sum_{C_{1}} \text {. } \\
& \sum_{C_{1}}=\sum_{\substack{t_{1} t_{2} \in V\left(H_{2}\right) \\
s_{1} s_{2} \in E\left(s_{k}\left(H_{1}\right)\right) s_{1} \in V\left(H_{1}\right), s_{2} \in V\left(s_{k}\left(H_{1}\right)-H_{1}\right)}}\left[\left(d\left(s_{1}\right)+d\left(t_{1}\right)+d\left(s_{1}\right) d\left(t_{1}\right)\right)^{2}+\left(d\left(s_{2}\right)+d\left(s_{2}\right) d\left(t_{2}\right)\right)^{2}\right] \\
& =\sum_{t \in V\left(H_{2}\right)} \sum_{\substack{s_{1} s_{2} \in E\left(s_{k}\left(H_{1}\right)\right) s_{1} \in V\left(H_{1}\right), s_{2} \in V\left(S_{k}\left(H_{1}\right)-H_{1}\right)}}\left[d\left(s_{1}\right)^{2}+d\left(s_{2}\right)^{2}+d\left(t_{1}\right)^{2} d\left(s_{1}\right)^{2}+d\left(t_{2}\right)^{2} d\left(s_{2}\right)^{2}\right] \\
& \left.+2 d\left(t_{1}\right) d\left(s_{1}\right)^{2}+2 d\left(t_{2}\right) d\left(s_{2}\right)^{2}+2 d\left(t_{1}\right)\left(d\left(s_{1}\right)+2 d^{2}\left(t_{1}\right)\right)\left(d\left(s_{1}\right)+d_{H_{2}}^{2}\left(t_{1}\right)\right)\right] \\
& =2\left|E\left(H_{2}\right)\right|\left|F\left(G_{1}\right)+16\right| E\left(H_{1}\right)|| E\left(H_{2}\right)|+2| E\left(H_{1}\right) \mid F\left(G_{2}\right)+F\left(G_{1}\right) F\left(G_{2}\right)+2 M_{1}\left(H_{1}\right) M_{1}\left(H_{2}\right) \\
& +2 F\left(G_{1}\right) M_{1}\left(H_{2}\right)+2 M_{1}\left(H_{1}\right) F\left(G_{2}\right)+8\left|E\left(H_{1}\right)\right| F\left(G_{2}\right)+16\left|E\left(H_{1}\right)\right| M_{1}\left(H_{2}\right), \\
& \sum_{C_{2}}=\sum_{t_{1} t_{2} \in E\left(H_{2}\right)} \sum_{s_{1} s_{2} \in E\left(S_{k}\left(H_{1}\right)\right) s_{1},}\left[\left(d\left(s_{1}\right)+d\left(s_{1}\right) d\left(t_{1}\right)\right)^{2}+\left(d\left(s_{2}\right)+d\left(s_{2}\right) d\left(t_{2}\right)\right)^{2}\right] \\
& s_{2} \in V\left(S_{k}\left(H_{1}\right)-H_{1}\right) \\
& =\sum_{t_{1} t_{2} \in E\left(H_{2}\right)} \sum_{\substack{s_{1} s_{2} \in E \\
s_{2} \in V\left(S_{k}\left(H_{1}\right)\right) s_{1},}}\left[8+4\left(d^{2}\left(t_{1}\right)+d^{2}\left(t_{2}\right)\right)+8\left(d\left(t_{1}\right)+d\left(t_{2}\right)\right)\right] \\
& =16(k-1)\left|E\left(H_{2}\right)\left\|E\left(H_{1}\right)\right\|\right|+8(k-1) F\left(H_{2}\right)\left|E\left(H_{1}\right)\right|+16(k-1)\left|E\left(H_{1}\right)\right| M_{1}\left(H_{2}\right) \text {. }
\end{aligned}
$$

We arrive at our desired result.

Theorem 2. Let $H_{1}$ and $H_{2}$ be two connected graphs, then 


$$
\begin{aligned}
& \left.F\left(H_{1_{R_{k}}} \otimes H_{2}\right)=8\left[\left|V\left(H_{2}\right)\right|+6\left|E\left(H_{2}\right)\right|\right] \mid\right] F\left(H_{1}\right)+\left[\left|V\left(H_{1}\right)\right|+20 \mid E\left(H_{1}\right)\right] F\left(H_{2}\right)+8 F\left(H_{1}\right) F\left(H_{2}\right) \\
& \quad+24\left|E\left(H_{2}\right)\right| M_{1}\left(H_{1}\right)+36\left|E\left(H_{1}\right)\right| M_{1}\left(H_{2}\right)+24 M_{1}\left(H_{1}\right) M_{1}\left(H_{2}\right)+24 F\left(H_{1}\right) M_{1}\left(H_{2}\right) \\
& \quad+8\left|V\left(H_{2}\right)\left\|E\left(H_{1}\right)\right\|\right|+8(k-1)\left|E\left(H_{1}\right)\right|\left[\left|V\left(H_{2}\right)\right|+F\left(H_{2}\right)+4\left|E\left(H_{2}\right)\right|+3 M_{1}\left(H_{2}\right)\right] \\
& \quad+48\left|E\left(H_{1}\right)\right|\left|E\left(H_{2}\right)\right|+12 F\left(H_{2}\right) M_{1}\left(H_{1}\right) .
\end{aligned}
$$

Proof. Let $d(s, t)=d_{H_{1_{R}} \otimes H_{2}}(s, t)$ is the degree of the vertex $(s, t)$ in $H_{1_{R_{k}}} \otimes H_{2}$, then

$$
\begin{aligned}
& F\left(H_{1_{R_{k}}} \otimes H_{2}\right)=\sum_{(s, t) \varepsilon V\left(H_{1_{R_{k}}} \otimes H_{2}\right)} d(s, t)^{3}=\sum_{\left(s_{1}, t_{1}\right)\left(s_{2}, t_{2}\right) \varepsilon E\left(H_{1_{R_{k}}} \otimes H_{2}\right)}\left[d\left(s_{1}, t_{1}\right)^{2}+d\left(s_{2}, t_{2}\right)^{2}\right] \\
& =\sum_{s \in V\left(H_{1}\right)} \sum_{t_{1} t_{2} E\left(H_{2}\right)}\left[d\left(s, t_{1}\right)^{2}+d\left(s, t_{2}\right)^{2}\right]+\sum_{t \in V\left(H_{2}\right)} \sum_{s_{1} s_{2} \in E\left(R_{k}\left(H_{1}\right)\right)}\left[d\left(s_{1}, t\right)^{2}+d\left(s_{2}, t\right)^{2}\right] \\
& \left.+\sum_{t_{1} t_{2} \in E\left(H_{2}\right)} \sum_{s_{1} s_{2} \in E}\left[d\left(R_{k}\left(H_{1}\right)\right), t_{1}\right)^{2}+d\left(s_{2}, t_{2}\right)^{2}\right]=\sum_{A}+\sum_{B}+\sum_{C} . \\
& \sum_{A}=\sum_{s e V\left(H_{1}\right)} \sum_{t_{1} t_{2} \in E\left(H_{2}\right)}\left[d\left(s, t_{1}\right)^{2}+d\left(s, t_{2}\right)^{2}\right]=8\left|E\left(H_{2}\right)\right| M_{1}\left(H_{1}\right)+\left|V\left(H_{1}\right)\right| F\left(H_{2}\right) \\
& +4 M_{1}\left(H_{1}\right) F\left(H_{2}\right)+8\left|E\left(H_{1}\right)\right| M_{1}\left(H_{2}\right)+8 M_{1}\left(H_{1}\right) M_{1}\left(H_{2}\right)+8\left|E\left(H_{1}\right)\right| F\left(H_{2}\right), \\
& \sum_{B}=\sum_{t \in V\left(H_{2}\right)} \sum_{\substack{s_{1} s_{2} \in V\left(H_{1}\right), s_{1}, s_{2} \in V\left(H_{1}\right)}}\left[d^{2}\left(s_{1}, t\right)+d^{2}\left(s_{2}, t\right)\right]+\sum_{\substack{t \in V\left(H_{2}\right) \\
s_{1} \in V}} \sum_{\substack{s_{2} \in V \\
s_{2} \in V \\
\left(R_{k}\left(H_{1}\right)-H_{1}\right)}}\left[d^{2}\left(s_{1}, t\right)+d^{2}\left(s_{2}, t\right)\right] \\
& \left.+\sum_{t \in V\left(H_{2}\right)} \sum_{s_{1}, s_{2} \in V}\left[R_{k}\left(R_{k}\left(H_{1}\right)-H_{1}\right) t\right)+d^{2}\left(s_{2}, t\right)\right]=\sum_{B_{1}}+\sum_{B_{2}}+\sum_{B_{3}} . \\
& \sum_{B_{1}}=\sum_{\operatorname{teV}\left(H_{2}\right)} \sum_{\substack{s_{1} s_{2} \in E\left(R_{k}\left(H_{1}\right)\right) s_{1}, s_{2} E V\left(H_{1}\right)}}\left[\left(d\left(s_{1}\right)+d(t)+d\left(s_{1}\right) d(t)\right)^{2}+\left(d\left(s_{2}\right)+d(t)+d\left(s_{2}\right) d(t)\right)^{2}\right] \\
& =\sum_{t \in V\left(H_{2}\right)} \sum_{s_{1} s_{2} E E\left(s_{k}\left(H_{1}\right)\right) s_{1},}\left[4\left(d^{2}\left(s_{1}\right)+d^{2}\left(s_{2}\right)\right)+2 d^{2}(t)+4 d^{2}(t)\left(d^{2}\left(s_{1}\right)+d^{2}\left(s_{2}\right)\right)\right] \\
& \left.\left.+4 d(t)\left(d\left(s_{1}\right)+d\left(s_{2}\right)\right)+4 d^{2}(t) d\left(s_{1}\right)+d\left(s_{2}\right)\right)+8 d(t)\left(d^{2}\left(s_{1}\right)+d^{2}\left(s_{2}\right)\right)\right] \\
& =4\left|V\left(H_{2}\right)\right| F\left(H_{1}\right)+2\left|E\left(H_{1}\right)\right| M_{1}\left(H_{2}\right)+4 M_{1}\left(H_{2}\right) F\left(H_{1}\right)+8\left|E\left(H_{2}\right)\right| M_{1}\left(H_{1}\right) \\
& +4 M_{1}\left(H_{1}\right) M_{1}\left(H_{2}\right)+16\left|E\left(H_{2}\right)\right| F\left(H_{1}\right) \\
& \sum_{B_{2}}=\sum_{t \in V\left(H_{2}\right)} \sum_{\substack{s_{1} s_{2} \in E\left(R_{k}\left(H_{1}\right)\right) s_{1} \in V\left(H_{1}\right), s_{2} \in V\left(R_{k}\left(H_{1}\right)-H_{1}\right)}}\left[\left(d\left(s_{1}\right)+d(t)+d\left(s_{1}\right) d(t)\right)^{2}+\left(d\left(s_{2}\right)+d\left(s_{2}\right) d(t)\right)^{2}\right] \\
& =\sum_{t \in V\left(H_{2}\right)} \sum_{\substack{s_{1} s_{2} \in E\left(R_{k}\left(H_{1}\right)\right) s_{1} \in V\left(H_{1}\right), s_{2} \in V\left(R_{k}\left(H_{1}\right)-H_{1}\right)}}\left[4 d\left(s_{1}\right)^{2}+d\left(s_{2}\right)^{2}+d(t)^{2}\left(4 d\left(s_{1}\right)^{2}+d\left(s_{2}\right)^{2}\right)\right] \\
& +8 d(t)\left(d\left(s_{1}\right)^{2}+4 d(t) d\left(s_{1}\right)+4 d^{2}(t)\left(d\left(s_{1}\right)+d^{2}(t)+2 d\left(s_{2}\right)^{2} d(t)\right)\right] \\
& =4\left|V\left(H_{2}\right)\right| F\left(H_{1}\right)+10\left|E\left(H_{1}\right)\right| M_{1}\left(H_{2}\right)+4 M_{1}\left(H_{2}\right) F\left(H_{1}\right)+8\left|E\left(H_{2}\right)\right| M_{1}\left(H_{1}\right) a \\
& +4 M_{1}\left(H_{1}\right) M_{1}\left(H_{2}\right)+16\left|E\left(H_{2}\right)\right| F\left(H_{1}\right)+8\left|V\left(H_{2}\right)\right|\left|E\left(H_{1}\right)\right|+32\left|E\left(H_{1}\right)\right|\left|E\left(H_{2}\right)\right|, \\
& \sum_{B_{3}}=\sum_{t \in V\left(H_{2}\right)} \sum_{\substack{s_{1} s_{2} \in E\left(R_{k}\left(H_{1}\right)\right) s_{1}, s_{2} E V\left(R_{k}\left(H_{1}\right)-H_{1}\right)}}\left[\left(d\left(s_{1}\right)+d\left(s_{1}\right) d(t)\right)^{2}+\left(d\left(s_{2}\right)+d\left(s_{2}\right) d(t)\right)^{2}\right] \\
& =\sum_{\operatorname{teV}\left(H_{2}\right)} \sum_{\substack{s_{1} s_{2} E E\left(R_{k}\left(H_{1}\right)\right) s_{1}, s_{2} E V\left(R_{k}\left(H_{1}\right)-H_{1}\right)}}\left[\left(d\left(s_{1}\right)^{2}+d\left(s_{2}\right)^{2}\right)+d(t)^{2}\left(d\left(s_{1}\right)^{2}+d\left(s_{2}\right)^{2}\right)+2 d(t)\left(d^{2}\left(s_{1}\right)+d^{2}\left(s_{2}\right)\right)\right]
\end{aligned}
$$




$$
\begin{aligned}
& =8(k-1) \mid\left(V\left(H_{2}\right)|| E\left(H_{1}\right)\left|+8(k-1) M_{1}\left(H_{2}\right)\right| E\left(H_{1}\right)|+32(k-1)| E\left(H_{2}\right)|| E\left(H_{1}\right) \mid,\right. \\
& \sum_{C}=\sum_{t_{1} t_{2} \in E\left(H_{2}\right)} \sum_{s_{1} s_{2} \in V\left(H_{1}\right)}\left[d^{2}\left(s_{1}, t_{1}\right)+d^{2}\left(s_{2}, t_{2}\right)\right]+\sum_{t_{1} t_{2} \in E\left(H_{2}\right)} \sum_{\substack{s_{1} \in V\left(H_{1}\right), s_{2} \in V\left(R_{k}\left(H_{1}\right)-H_{1}\right)}}\left[d^{2}\left(s_{1}, t_{1}\right)+d^{2}\left(s_{2}, t_{2}\right)\right] \\
& \left.\left.+\sum_{t_{1} t_{2} \in E\left(H_{2}\right)} \sum_{s_{1}, s_{2} \in V}\left[d^{2}\left(s_{1}, t_{1}\right)+d^{2}\left(H_{1}\right)-H_{1}\right) t_{2}\right)\right]=\sum_{C_{1}}+\sum_{C_{2}}+\sum_{C_{3}} . \\
& \sum_{C_{1}}=\sum_{t_{1} t_{2} \in V\left(H_{2}\right)} \sum_{\substack{s_{1} s_{2} \in E\left(R_{k}\left(H_{1}\right)\right) s_{1}, s_{2} \in V\left(H_{1}\right)}}\left[\left(d\left(s_{1}\right)+d\left(t_{1}\right)+d\left(s_{1}\right) d\left(t_{1}\right)\right)^{2}+\left(d\left(s_{2}\right)+d\left(t_{2}\right)+d\left(s_{2}\right) d\left(t_{2}\right)\right)^{2}\right] \\
& =\sum_{t_{1} t_{2} \in V\left(H_{2}\right)} \sum_{\substack{s_{1} s_{2} \in E\left(R_{k}\left(H_{1}\right)\right) s_{1}, s_{2} \in V\left(H_{1}\right)}}\left[d\left(s_{1}\right)^{2}+d\left(s_{2}\right)^{2}+d^{2}\left(t_{1}\right)+d^{2}\left(t_{2}\right)+d\left(t_{1}\right)^{2} d\left(s_{1}\right)^{2}+d^{2}\left(t_{2}\right) d\left(s_{2}\right)^{2}+2 d\left(t_{1}\right) d\left(s_{1}\right)\right] \\
& \left.+2 d\left(t_{2}\right) d\left(s_{2}\right)+2 d^{2}\left(t_{1}\right) d\left(s_{1}\right)+2 d^{2}\left(t_{2}\right) d\left(s_{2}\right)+2 d\left(t_{1}\right) d^{2}\left(s_{1}\right)+2 d^{2}\left(t_{2}\right) d\left(s_{2}\right)\right] \\
& =8\left|E\left(H_{2}\right)\right| F\left(H_{1}\right)+2\left|E\left(H_{1}\right)\right| F\left(H_{2}\right)+4 F\left(H_{1}\right) F\left(H_{2}\right)+4 M_{1}\left(H_{1}\right) M_{1}\left(H_{2}\right) \\
& +4 M_{1}\left(H_{1}\right) F\left(H_{2}\right)+8 M_{1}\left(H_{2}\right) F\left(H_{1}\right) \\
& \sum_{C_{2}}=\sum_{\substack{t_{1} t_{2} \in V\left(H_{2}\right) \\
s_{1} s_{2} \in E\left(R_{k}\left(H_{1}\right)\right) s_{1} \in V\left(H_{1}\right), s_{2} \in V\left(R_{k}\left(H_{1}\right)-H_{1}\right)}}\left[\left(d\left(s_{1}\right)+d\left(t_{1}\right)+d\left(s_{1}\right) d\left(t_{1}\right)\right)^{2}+\left(d\left(s_{2}\right)+d\left(s_{2}\right) d\left(t_{2}\right)\right)^{2}\right] \\
& =\sum_{t \varepsilon V\left(H_{2}\right)} \sum_{\substack{s_{1} s_{2} \varepsilon E\left(R_{k}\left(H_{1}\right)\right) s_{1} \varepsilon V\left(H_{1}\right), s_{2} \varepsilon V\left(R_{k}\left(H_{1}\right)-H_{1}\right)}}\left[d\left(s_{1}\right)^{2}+d\left(s_{2}\right)^{2}+d^{2}\left(t_{1}\right)+d\left(t_{1}\right)^{2} d\left(s_{1}\right)^{2}+2 d\left(t_{1}\right) d\left(s_{1}\right)+2 d\left(t_{1}\right) d\left(s_{1}\right)^{2}\right] \\
& \left.+2 d^{2}\left(t_{1}\right) d\left(s_{1}\right)+d\left(t_{2}\right)^{2} d\left(s_{2}\right)^{2}+2 d\left(t_{2}\right) d\left(s_{2}\right)^{2}\right] \\
& =8\left|E\left(H_{2}\right)\right| F\left(G_{1}\right)+16\left|E\left(H_{1}\right)\right|\left|E\left(H_{2}\right)\right|+4 F\left(G_{1}\right) F\left(G_{2}\right)+4 M_{1}\left(H_{1}\right) M_{1}\left(H_{2}\right)+8 F\left(G_{1}\right) M_{1}\left(H_{2}\right) \\
& +2\left|E\left(H_{1}\right)\right| F\left(G_{2}\right)+4 M_{1}\left(H_{1}\right) F\left(G_{2}\right)+8\left|E\left(H_{1}\right)\right| F\left(G_{2}\right)+16\left|E\left(H_{1}\right)\right| M_{1}\left(H_{2}\right) \text {, } \\
& \sum_{C_{3}}=\sum_{t_{1} t_{2} \in E\left(H_{2}\right)} \sum_{\substack{s_{1} s_{2} \in E\left(R_{k}\left(H_{1}\right)\right) s_{1}, s_{2} \in V\left(R_{k}\left(H_{1}\right)-H_{1}\right)}}\left[\left(d\left(s_{1}\right)+d\left(s_{1}\right) d\left(t_{1}\right)\right)^{2}+\left(d\left(s_{2}\right)+d\left(s_{2}\right) d\left(t_{2}\right)\right)^{2}\right] \\
& =\sum_{t_{1} t_{2} \in E\left(H_{2}\right)} \sum_{\substack{s_{1} s_{2} \in E\left(R_{k}\left(H_{1}\right)\right) s_{1}, s_{2} \in V\left(R_{k}\left(H_{1}\right)-H_{1}\right)}}\left[\left(2+2 d\left(t_{1}\right)\right)^{2}+\left(2+2 d\left(t_{2}\right)\right)^{2}\right] \\
& =16(k-1)\left|E\left(H_{2}\right)\right|\left|E\left(H_{1}\right)\right|+8(k-1) F\left(H_{2}\right)\left|E\left(H_{1}\right)\right|+16(k-1)\left|E\left(H_{1}\right)\right| M_{1}\left(H_{2}\right) \text {. }
\end{aligned}
$$

We arrive at our desired result.

Theorem 3. Let $H_{1}$ and $H_{2}$ be two connected graphs, then

$$
\begin{aligned}
& F\left(H_{1_{Q_{k}}} \otimes H_{2}\right)=2(k-1)\left[F\left(H_{1}\right)+2 M_{2}\left(H_{1}\right)\right]\left[3 n\left(H_{2}\right)+5 M_{1}\left(H_{2}\right)+14\left|E\left(H_{2}\right)\right|+F\left(H_{2}\right)\right]+k\left[\left|V\left(H_{2}\right)\right|\right. \\
& \left.\quad+6\left|E\left(H_{2}\right)\right|+3 M_{1}\left(H_{2}\right)+F\left(H_{2}\right)\right]\left[M_{4}\left(H_{1}\right)-2 F\left(H_{1}\right)+2 M_{2}\left(H_{1}\right)-4 M_{2}\left(H_{1}\right)\right. \\
& \left.\quad+\sum_{u \in V\left(H_{1}\right)} d^{2}(u) \sum_{v \in N(u)} d(v)\right]+6\left|E\left(H_{2}\right)\right| M_{1}\left(H_{1}\right)+10\left|E\left(H_{2}\right)\right| F\left(H_{2}\right)+3 F\left(H_{1}\right) F\left(H_{2}\right) \\
& \quad+6 M_{1}\left(H_{1}\right) M_{1}\left(H_{2}\right)+F\left(H_{2}\right)\left[\left|V\left(H_{1}\right)\right|+3 M_{1}\left(H_{1}\right)+6 \mid E\left(H_{2}\right)+4 M_{2}\left(H_{1}\right)\right] \\
& \quad+F\left(H_{1}\right)\left[\left|V\left(H_{2}\right)\right|+7 M_{1}\left(H_{2}\right)\right]+6\left|E\left(H_{2}\right)\right| M_{2}\left(H_{1}\right)+8 M_{2}\left(H_{1}\right)\left[\left|E\left(H_{2}\right)\right|+M_{1}\left(H_{2}\right)\right]
\end{aligned}
$$

Proof. Let $d(s, t)=d_{H_{1} \otimes H_{2}}(s, t)$ is the degree of the vertex $(s, t)$ in $H_{1_{Q_{k}}} \otimes H_{2}$, then 


$$
\begin{aligned}
& F\left(H_{1_{Q_{k}}} \otimes H_{2}\right)=\sum_{(s, t) \in V\left(H_{Q_{Q_{k}}} \otimes H_{2}\right)} d(s, t)^{3}=\sum_{\left(s_{1}, t_{1}\right)\left(s_{2}, t_{2}\right) \in E\left(H_{Q_{Q_{k}}} \otimes H_{2}\right)}\left[d\left(s_{1}, t_{1}\right)^{2}+d\left(s_{2}, t_{2}\right)^{2}\right] \\
& =\sum_{s \in V\left(H_{1}\right)} \sum_{t_{1} t_{2} \in E\left(H_{2}\right)}\left[d\left(s, t_{1}\right)^{2}+d\left(s, t_{2}\right)^{2}\right]+\sum_{t \in V\left(H_{2}\right)} \sum_{s_{1} s_{2} \in E\left(Q_{k}\left(H_{1}\right)\right)}\left[d\left(s_{1}, t\right)^{2}+d\left(s_{2}, t\right)^{2}\right] \\
& +\sum_{t_{1} t_{2} \in E\left(H_{2}\right)} \sum_{s_{1} s_{2} \in E\left(Q_{k}\left(H_{1}\right)\right)}\left[d\left(s_{1}, t_{1}\right)^{2}+d\left(s_{2}, t_{2}\right)^{2}\right]=\sum_{A}+\sum_{B}+\sum_{C} . \\
& \sum_{A}=\sum_{s \in V\left(H_{1}\right)} \sum_{t_{1} t_{2} \in E\left(H_{2}\right)}\left[d\left(s, t_{1}\right)^{2}+d\left(s, t_{2}\right)^{2}\right]=2\left|E\left(H_{2}\right)\right| M_{1}\left(H_{1}\right)+\left|V\left(H_{1}\right)\right| F\left(H_{2}\right) \\
& +M_{1}\left(H_{1}\right) F\left(H_{2}\right)+4\left|E\left(H_{1}\right)\right| M_{1}\left(H_{2}\right)+2 M_{1}\left(H_{1}\right) M_{1}\left(H_{2}\right)+4\left|E\left(H_{1}\right)\right| F\left(H_{2}\right) . \\
& \sum_{B}=\sum_{t \in V\left(H_{2}\right)} \sum_{s_{1} s_{2} \in E\left(Q_{k}\left(H_{1}\right)\right)}\left[d^{2}\left(s_{1}, t\right)+d^{2}\left(p_{2}, t\right)\right]=\sum_{t \in V\left(H_{2}\right)} \sum_{\substack{s_{1} \in V\left(H_{1}\right), s_{2} \in V\left(Q_{k}\left(H_{1}\right)-H_{1}\right)}}\left[d^{2}\left(s_{1}, t\right)+d^{2}\left(s_{2}, t\right)\right]
\end{aligned}
$$

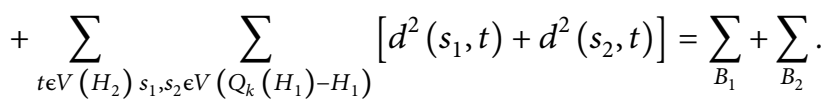

$$
\begin{aligned}
& \sum_{B_{1}}=\sum_{t \in V\left(H_{2}\right)} \sum_{s_{1} s_{2} \in E\left(Q_{k}\left(H_{1}\right)\right) s_{1} \in V\left(H_{1}\right), s}\left[\left(d\left(s_{1}\right)+d(t)+d\left(s_{1}\right) d(t)\right)^{2}+\left(d\left(s_{2}\right)+d\left(s_{2}\right) d(t)\right)^{2}\right] \\
& =\sum_{t \varepsilon V\left(H_{2}\right)} \sum_{\substack{s_{1} s_{2} \varepsilon E\left(Q_{k}\left(H_{1}\right)\right) s_{1} \varepsilon V\left(H_{1}\right), s_{2} \varepsilon V\left(Q_{k}\left(H_{1}\right)-H_{1}\right)}}\left[d\left(s_{1}\right)^{2}+d\left(s_{2}\right)^{2}+d(t)^{2}\left(d\left(s_{1}\right)^{2}+d\left(s_{2}\right)^{2}\right)+2 d(t) d\left(s_{1}\right)^{2}+2 d(t) d^{2}\left(s_{2}\right)^{2}\right] \\
& \left.+2 d(t) d^{2}\left(s_{2}\right)^{2}+2 d(t) d\left(s_{1}\right)+d^{2}(t)\right] \text {. }
\end{aligned}
$$

Consider $s_{1} \in V\left(H_{1}\right)$ and $d^{2}\left(s_{1}\right)$ occurs $d\left(s_{1}\right)$ times. Thus,

$$
D_{1}=\sum_{\substack{s_{1} s_{2} \in E\left(Q\left(H_{1}\right)\right), s_{1} \varepsilon V\left(H_{1}\right), s_{2} \varepsilon V\left(Q\left(H_{1}\right)\right)-V\left(H_{1}\right)}} d^{3}\left(s_{1}\right)=F\left(H_{1}\right) .
$$

$$
D_{2}=\sum_{\substack{s_{1} s_{2} \in E\left(Q\left(H_{1}\right)\right), s_{1} \varepsilon V\left(H_{1}\right), s_{2} \in V\left(Q\left(H_{1}\right)\right)-V\left(H_{1}\right)}} d^{2}\left(s_{2}\right) .
$$

Let

$$
\begin{aligned}
& D_{2}=2 \sum_{s_{2}=u v \in V\left(Q\left(H_{1}\right)\right)-V\left(H_{1}\right)}[d(u)+d(v)]^{2}=2 \sum_{u v \in E\left(H_{1}\right)}\left[d^{2}(u)+d^{2}(v)+2 d(u) d(v)\right]=2\left[F\left(H_{1}\right)+2 M_{2}\left(H_{1}\right)\right] \\
& =\left|V\left(H_{2}\right)\right| F\left(H_{1}\right)+2\left|E\left(H_{1}\right)\right| M_{1}\left(H_{2}\right)+M_{1}\left(H_{2}\right) F\left(H_{1}\right)+4\left|E\left(H_{2}\right)\right|\left[M_{1}\left(H_{1}\right)+F\left(H_{1}\right)\right]+2 M_{1}\left(H_{1}\right) M_{1}\left(H_{2}\right) \\
& \quad+2\left|V\left(H_{2}\right)\right|\left[F\left(H_{1}\right)+2 M_{2}\left(H_{1}\right)\right]+2 M_{1}\left(H_{2}\right)\left[F\left(H_{1}\right)+2 M_{2}\left(H_{1}\right)\right]+8\left|E\left(H_{2}\right)\right|\left[F\left(H_{1}\right)+2 M_{2}\left(H_{1}\right)\right] . \\
& \sum_{B_{2}}=\sum_{t \in V\left(H_{2}\right)} \sum_{s_{1} s_{2} \in E\left(Q_{k}\left(H_{1}\right)\right) s_{s_{1}, s_{2} \in V}\left(Q_{k}\left(H_{1}\right)-H_{1}\right)}\left[\left(d\left(s_{1}\right)+d\left(s_{1}\right) d(t)\right)^{2}+\left(d\left(s_{2}\right)+d\left(s_{2}\right) d(t)\right)^{2}\right] .
\end{aligned}
$$

Now, we split sum in two parts, $s_{1}$ and $s_{2}$, where $s_{1} s_{2} \epsilon$ $V\left(Q_{k}\left(H_{1}\right)\right)-V\left(H_{1}\right)$. Suppose that $\sum_{B_{2}}=\sum B_{21}+\sum B_{22}$, where $\sum B_{21}$ covers the edges of $Q_{k}\left(H_{1}\right)$ which are in the same edges of $H_{1}$ and $\sum B_{22}$ of $Q_{k}\left(H_{1}\right)$ in two different adjacent edges of $H_{1}$. 


$$
\begin{aligned}
\sum_{B_{21}} & =\sum_{t \in V\left(H_{1}\right)} \sum_{s_{1}, s_{2} E E\left(Q\left(H_{1}\right)\right)}\left[\left\{d\left(s_{1}\right)+d\left(s_{1}\right) d(t)\right\}^{2}+\left\{d\left(s_{2}\right)+d\left(s_{2}\right) d(t)\right\}^{2}\right] \\
& =\sum_{t \in V\left(H_{2}\right)} \sum_{s_{1}, s_{2} E E\left(Q\left(H_{1}\right)\right)}\left[\left[d^{2}\left(s_{1}\right)+d^{2}\left(s_{2}\right)\right]+d^{2}(t)\left[d^{2}\left(s_{1}\right)+d^{2}\left(s_{2}\right)\right]+2 d(t)\left[d^{2}\left(s_{1}\right)+d^{2}\left(s_{2}\right)\right]\right] \\
& =\sum_{t \in V\left(H_{2}\right)} 2(k-1)\left[\left[F\left(H_{1}\right)+2 M_{2}\left(H_{1}\right)\right]+d^{2}(t)\left[F\left(H_{1}\right)+2 M_{2}\left(H_{1}\right)\right]+2 d(t)\left[F\left(H_{1}\right)+2 M_{2}\left(H_{1}\right)\right]\right. \\
& =2(k-1)\left[F\left(H_{1}\right)+2 M_{2}\left(H_{1}\right)\right]\left[\left|V\left(H_{2}\right)\right|+M_{1}\left(H_{2}\right)+4\left|E\left(H_{2}\right)\right|\right], \\
\sum_{B_{22}} & =\sum_{t \in V\left(H_{2}\right)} \sum_{\substack{s_{1} s_{2} \in E\left(Q_{k}\left(H_{1}\right)\right) s_{1}, s_{2} \in V\left(Q_{k}\left(H_{1}\right)-H_{1}\right)}}\left[d\left(s_{1}\right)^{2}+d\left(s_{2}\right)^{2}+d(t)^{2}\left(d\left(s_{1}\right)^{2}+d\left(s_{2}\right)^{2}\right)+2 d(t)\left(d\left(s_{1}\right)^{2}+d\left(s_{2}\right)^{2}\right)\right] \\
D_{3}= & \sum_{\substack{s_{1} s_{2} \in E\left(Q\left(H_{1}\right)\right) s_{1}, s_{2} \in V\left(Q\left(H_{1}\right)\right)-V\left(H_{1}\right)}}\left[d^{2}\left(s_{1}\right)+d^{2}\left(s_{2}\right)\right] .
\end{aligned}
$$

In $D_{3}$, the coefficient of

$$
\begin{aligned}
d^{2}(u) & =2\left(\begin{array}{c}
2 \\
d\left(H_{1}\right)(u)
\end{array}\right)+\sum_{v \in N(u)} d(v)-d(u) \\
& =d^{2}(u)-2 d(u)+\sum_{v \in N(u)} d(v) .
\end{aligned}
$$

Therefore,

$$
\begin{aligned}
\sum_{u \in V\left(H_{1}\right)} d^{2}(u) & =M_{4}\left(H_{1}\right)-2 F\left(H_{1}\right)+\sum_{u \in V\left(H_{1}\right)} d^{2}(u) \\
& \cdot \sum_{v \in N(u)} d(v) .
\end{aligned}
$$

For the coefficient of $\mathrm{d} u \mathrm{~d} v$, let $s_{1} s_{2} \in E\left(Q\left(H_{1}\right)\right)$ with $s_{1}=$ $u v$ and $s_{2}=w z$. As $s_{1} s_{2} \in E\left(Q\left(H_{1}\right)\right)$, we have either $v=w$ or $z$ or $u=w$ or $z$. So, $u v$ is adjacent to all those vertices in $H_{1}$ which are adjacent to $u$ and $v$. So, the number of such $\mathrm{d} u \mathrm{~d} v$ is $(\mathrm{d} u+\mathrm{d} v-2)$. Therefore,

$$
\begin{aligned}
& 2 \sum_{u v \in E\left(H_{1}\right)} \mathrm{d} u \mathrm{~d} v=2 \sum_{u v \in E\left(H_{1}\right)}(\mathrm{d} u+\mathrm{d} v-2) \mathrm{d} u \mathrm{~d} v \\
& =2 \sum_{u v \in E\left(H_{1}\right)}(\mathrm{d} u+\mathrm{d} v) \mathrm{d} u \mathrm{~d} v-4 \sum_{u v \in E\left(H_{1}\right)} \mathrm{d} u \mathrm{~d} v \\
& =2 M_{2}^{1}\left(H_{1}\right)-4 M_{2}\left(H_{1}\right) .
\end{aligned}
$$

So,

$$
\begin{aligned}
& D_{3}=M_{4}\left(H_{1}\right)-2 F\left(H_{1}\right)+\sum_{u \in V\left(H_{1}\right)} \mathrm{d}^{2}(u) \sum_{v \in N(u)} \mathrm{d}(v)+2 M_{2}^{1}\left(H_{1}\right)-4 M_{2}\left(H_{1}\right) \\
& \sum_{B_{22}}=(k)\left[\left|V\left(H_{2}\right)\right|+4\left|E\left(H_{2}\right)\right|+M_{1}\left(H_{2}\right)\right]\left[M_{4}\left(H_{1}\right)-2 F\left(H_{1}\right)+2 M_{2}^{1}\left(H_{1}\right)-4 M_{2}\left(H_{1}\right)\right. \\
& \left.+\sum_{u \in V\left(H_{1}\right)} \mathrm{d}^{2}(u) \sum_{v \in N(u)} \mathrm{d}(v)\right] \\
& \sum_{C}=\sum_{t_{1} t_{2} \in E\left(H_{2}\right)} \sum_{s_{1} s_{2} \in E}\left[Q_{k}\left(H_{1}\right)\right)\left(d^{2}\left(s_{1}, t_{1}\right)+d^{2}\left(s_{2}, t_{2}\right)\right]=\sum_{t_{1} t_{2} \in E\left(H_{2}\right)} \sum_{\substack{s_{1} \in V\left(H_{1}\right), s_{2} \in V\left(Q_{k}\left(H_{1}\right)-H_{1}\right)}}\left[d^{2}\left(s_{1}, t_{1}\right)+d^{2}\left(s_{2}, t_{2}\right)\right] \\
& \left.+\sum_{t_{1} t_{2} \in E\left(H_{2}\right)} \sum_{s_{1}, s_{2} \in V}\left[Q^{2}\left(s_{1}, t_{1}\right)+d^{2}\left(s_{1}\right)-H_{2}\right)\right]=\sum_{C_{1}}+\sum_{C_{2}} \text {. } \\
& \sum_{C_{1}}=\sum_{t_{1} t_{2} \in V\left(H_{2}\right)} \sum_{s_{1} s_{2} E E\left(Q_{k}\left(H_{1}\right)\right) s_{1} \in V\left(H_{1}\right),}\left[\left(d\left(s_{1}\right)+d\left(t_{1}\right)+d\left(s_{1}\right) d\left(t_{1}\right)\right)^{2}+\left(d\left(s_{2}\right)+d\left(s_{2}\right) d\left(t_{2}\right)\right)^{2}\right] \\
& =\sum_{t \in V\left(H_{2}\right)} \sum_{\substack{s_{1} s_{2} \in E\left(Q_{k}\left(H_{1}\right)\right) s_{1} \in V\left(H_{1}\right), s_{2} \in V\left(Q_{k}\left(H_{1}\right)-H_{1}\right)}}^{s_{2} \in V\left(Q_{k}\left(H_{1}\right)-H_{1}\right)}\left[d\left(s_{1}\right)^{2}+d\left(t_{1}\right)^{2} d\left(s_{1}\right)^{2}+2 d\left(t_{1}\right) d\left(s_{1}\right)+2 d^{2}\left(t_{1}\right) d\left(s_{1}\right)+2 d\left(t_{1}\right) d\left(s_{1}\right)^{2}\right] \\
& \left.+d^{2}\left(t_{1}\right)+d\left(s_{2}\right)^{2}+d\left(t_{2}\right)^{2} d\left(s_{2}\right)^{2}+2 d\left(t_{2}\right) d\left(s_{2}\right)^{2}\right] \\
& =6\left[\left|E\left(H_{2}\right)\right|+M_{1}\left(H_{2}\right)\right] F\left(H_{1}\right)+3 F\left(H_{1}\right) F\left(H_{2}\right)+2 M_{1}\left(H_{1}\right) M_{1}\left(H_{2}\right)+2\left[\left|E\left(H_{1}\right)\right|+M_{1}\left(H_{1}\right)\right. \\
& \left.+2 M_{2}\left(H_{1}\right)\right] F\left(H_{2}\right)+8 M_{2}\left(H_{1}\right)\left[\left|E\left(H_{2}\right)\right|+M_{1}\left(H_{2}\right)\right] . \mid \\
& \sum_{C_{2}}=\sum_{t_{1} t_{2} \in E\left(H_{2}\right)} \sum_{s_{1} s_{2} \in E\left(Q_{k}\left(H_{1}\right)\right) s_{1},}\left[\left(d\left(s_{1}\right)+d\left(s_{1}\right) d\left(t_{1}\right)\right)^{2}+\left(d\left(s_{2}\right)+d\left(s_{2}\right) d\left(t_{2}\right)\right)^{2}\right] \text {. } \\
& s_{2} \in V\left(Q_{k}\left(H_{1}\right)-H_{1}\right)
\end{aligned}
$$


TABle 1: $F$-index of $F_{1}$-sum path graphs.

\begin{tabular}{lcccc}
\hline$\left[n_{1}, n_{2}\right]$ & $F\left(P_{n_{1_{1}}} \otimes P_{n_{2}}\right)$ & $F\left(P_{n_{1_{1}}} \otimes P_{n_{2}}\right)$ & $F\left(P_{n_{1_{1}}} \otimes P_{n_{2}}\right)$ & $F\left(P_{n_{1_{1}}} \otimes P_{n_{2}}\right)$ \\
\hline$(3,3)$ & 1808 & 6414 & 3442 & 8048 \\
$(4,4)$ & 4836 & 18120 & 8826 & 22,110 \\
$(5,5)$ & 9320 & 35,746 & 16,692 & 43,118 \\
$(6,6)$ & 15,260 & 59,292 & 27,040 & 71,072 \\
$(7,7)$ & 22,656 & 88,758 & 39,870 & 105,972 \\
\hline
\end{tabular}

TABle 2: $F$-index of $F_{2}$-sum path graphs.

\begin{tabular}{lcccc}
\hline$\left[n_{1}, n_{2}\right]$ & $F\left(P_{n_{1_{S_{2}}}} \otimes P_{n_{2}}\right)$ & $F\left(P_{n_{1_{R_{2}}}} \otimes P_{n_{2}}\right)$ & $F\left(P_{n_{\mathrm{Q}_{2}}} \otimes P_{n_{2}}\right)$ & $F\left(P_{n_{1_{T_{2}}}} \otimes P_{n_{2}}\right)$ \\
\hline$(3,3)$ & 2496 & 7102 & 5764 & 10,370 \\
$(4,4)$ & 6516 & 19,800 & 14,496 & 27,780 \\
$(5,5)$ & 12,424 & 38,850 & 27,168 & 53,594 \\
$(6,6)$ & 20,220 & 64,252 & 43,780 & 87,812 \\
$(7,7)$ & 29,904 & 96,006 & 64,332 & 130,434 \\
\hline
\end{tabular}

Now, we split this sum into two parts for the vertices, $t_{1}$ and $t_{2}$, where $s_{1} s_{2} \in V\left(Q_{k}(G)-V(G)\right.$. Assume that (Tex translation failed), where (Tex translation failed) cover the edges of $Q_{k}\left(H_{1}\right)$ which are in the same edges of $H_{1}$ and (Tex translation failed) of $Q_{k}\left(H_{1}\right)$ in two different adjacent edges of $H_{1}$.

$$
\begin{aligned}
\sum_{C_{21}}= & \sum_{t_{1} t_{2} \in E\left(H_{2}\right)} \sum_{\substack{s_{1} s_{2} \in E\left(Q_{k}\left(H_{1}\right)\right) s_{1}, s_{2} \in V\left(Q_{k}\left(H_{1}\right)-H_{1}\right)}}\left[\left(d\left(s_{1}\right)+d\left(s_{1}\right) d\left(t_{1}\right)\right)^{2}+\left(d\left(s_{2}\right)+d\left(s_{2}\right) d\left(t_{2}\right)\right)^{2}\right] \\
= & \sum_{\substack{t_{1} t_{2} \in E\left(H_{2}\right) \\
s_{1} s_{2} \in E\left(Q_{k}\left(H_{1}\right)\right) s_{1}, s_{2} \in V\left(Q_{k}\left(H_{1}\right)-H_{1}\right)}}\left[d^{2}\left(s_{1}\right)+d^{2}\left(s_{2}\right)+d^{2}\left(s_{1}\right) d^{2}\left(t_{1}\right)+d^{2}\left(s_{2}\right) d^{2}\left(t_{2}\right)+2\left[d^{2}\left(s_{1}\right) d\left(t_{1}\right)+d^{2}\left(s_{2}\right) d\left(t_{2}\right)\right]\right] \\
= & 2(k-1)\left[F\left(H_{1}\right)+2 M_{2}\left(H_{1}\right)\right]\left[2\left|E\left(H_{2}\right)\right|+F\left(H_{2}\right)+2 M_{1}\left(H_{2}\right)\right] \\
\sum_{C_{22}=} & \sum_{t_{1} t_{2} \in E\left(H_{2}\right) s_{1} s_{s_{2} \in E\left(Q_{k}\left(H_{1}\right)\right) s_{1},}}\left[\left(d\left(s_{1}\right)+d\left(s_{1}\right) d\left(t_{1}\right)\right)^{2}+\left(d\left(s_{2}\right)+d\left(s_{2}\right) d\left(t_{2}\right)\right)^{2}\right] \\
= & (k)\left[2\left|E\left(H_{2}\right)\right|+F\left(H_{2}\right)+2 M_{1}\left(H_{2}\right)\right]\left(\left[M_{4}\left(H_{1}\right)-2 F\left(H_{1}\right)+2 M_{2}\left(H_{1}\right)-4 M_{2}\left(H_{1}\right)\right.\right. \\
& \left.+\sum_{u \in V\left(H_{1}\right)} d^{2}(u) \sum_{v \in N(u)} d(v)\right] .
\end{aligned}
$$

We arrive at our desired result.

Theorem 4. Let $H_{1}$ and $H_{2}$ be two connected graphs, then

$$
\begin{aligned}
F\left(H_{1_{T_{k}}} \otimes H_{2}\right)= & 2(k-1)\left[F\left(H_{1}\right)+2 M_{2}\left(H_{1}\right)\right]\left[n\left(H_{2}\right)+3 M_{1}\left(H_{2}\right)+6\left|E\left(H_{2}\right)\right|+F\left(H_{2}\right)\right]+k\left[\left|V\left(H_{2}\right)\right|\right. \\
& \left.+6\left|E\left(H_{2}\right)\right|+3 M_{1}\left(H_{2}\right)+F\left(H_{2}\right)\right]\left[M_{4}\left(H_{1}\right)-2 F\left(H_{1}\right)+2 M_{2}\left(H_{1}\right)-4 M_{2}\left(H_{1}\right)\right. \\
& \left.+\sum_{u \in V\left(H_{1}\right)} d^{2}(u) \sum_{v \in N(u)} d(v)\right]+\left[F\left(H_{1}\right)+2 M_{2}\left(H_{1}\right)\right]\left[2 n\left(H_{2}\right)+6 M_{1}\left(H_{2}\right)+12\left|E\left(H_{2}\right)\right|+2 F\left(H_{2}\right)\right] \\
& +4 F\left(H_{1}\right)\left[2 n\left(H_{2}\right)+6 M_{1}\left(H_{2}\right)+12\left|E\left(H_{2}\right)\right|+2 F\left(H_{2}\right)\right]+F\left(H_{2}\right)\left[n\left(H_{1}\right)+12 M_{1}\left(H_{1}\right)\right. \\
& \left.+12\left|E\left(H_{2}\right)\right|\right]+12\left|E\left(H_{1}\right)\right| M_{1}\left(H_{2}\right)+16\left|E\left(H_{2}\right)\right| M_{1}\left(H_{1}\right)+20 M_{1}\left(H_{1}\right) M_{1}\left(H_{2}\right) .
\end{aligned}
$$

Proof. It follows from Theorems 2 and 3. 


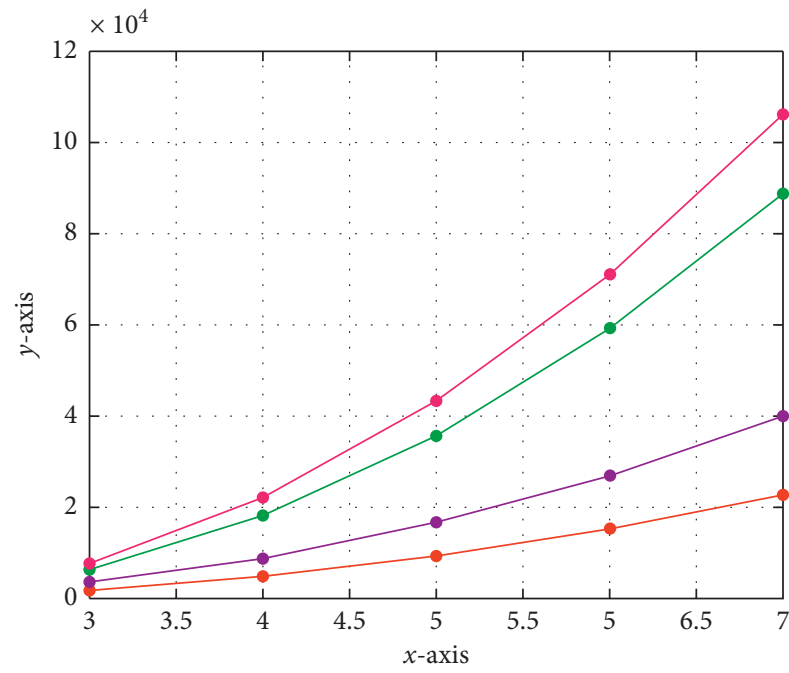

(a)

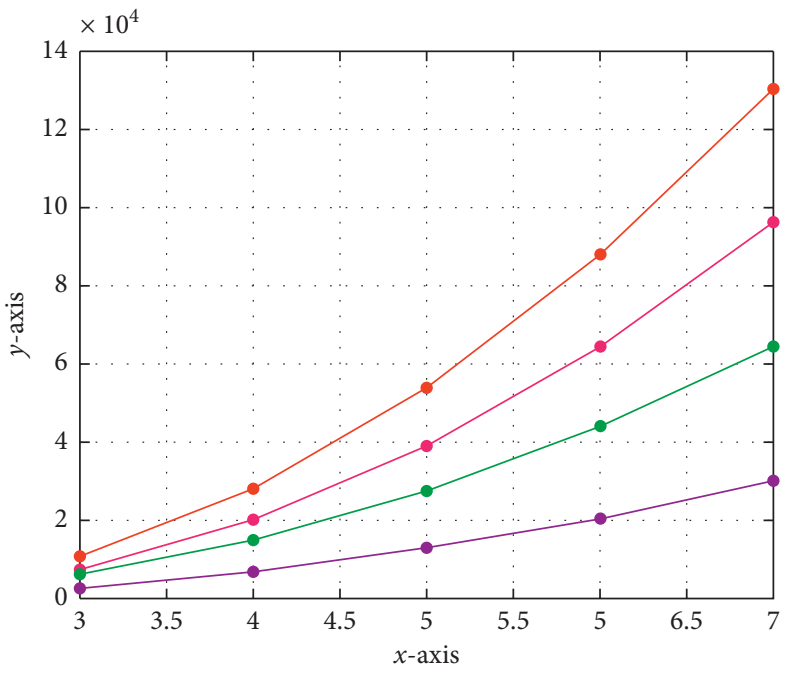

(b)

FIGURE 8: (a) $F\left(P_{n_{1_{1}}} \otimes P_{n_{2}}\right), F\left(P_{n_{1_{1}}} \otimes P_{n_{2}}\right), F\left(P_{n_{1_{R_{1}}}} \otimes P_{n_{2}}\right)$, and $F\left(P_{n_{1_{1}}} \otimes P_{n_{2}}\right)$ denoted by red, purple, green, and pink colours, respectively. (b) $F\left(P_{n_{s_{2}}} \otimes P_{n_{2}}\right), F\left(P_{n_{1_{Q_{2}}}} \otimes P_{n_{2}}\right), F\left(P_{n_{R_{2}}} \otimes P_{n_{2}}\right)$, and $F\left(P_{n_{1_{T_{2}}}} \otimes P_{n_{2}}\right)$ denoted by purple, green, pink, and red colours, respectively.

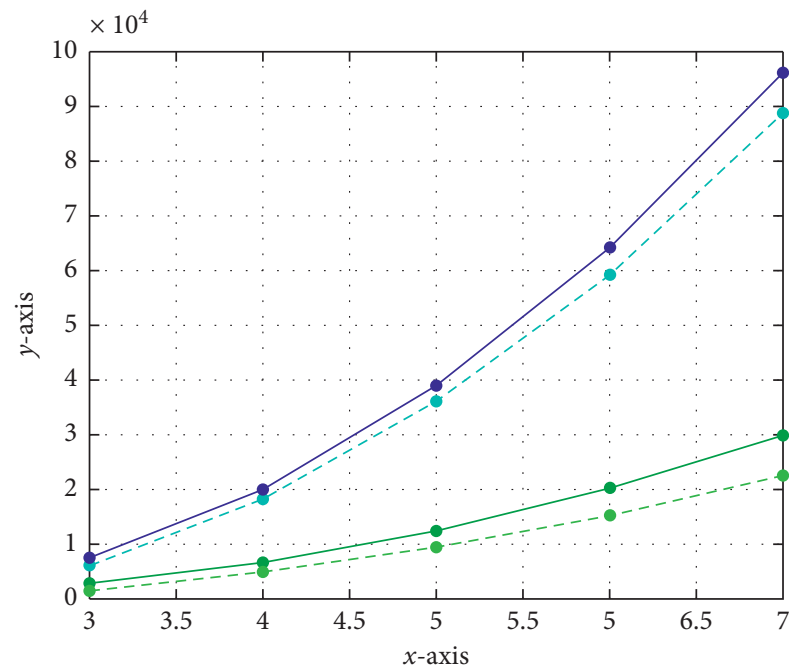

(a)

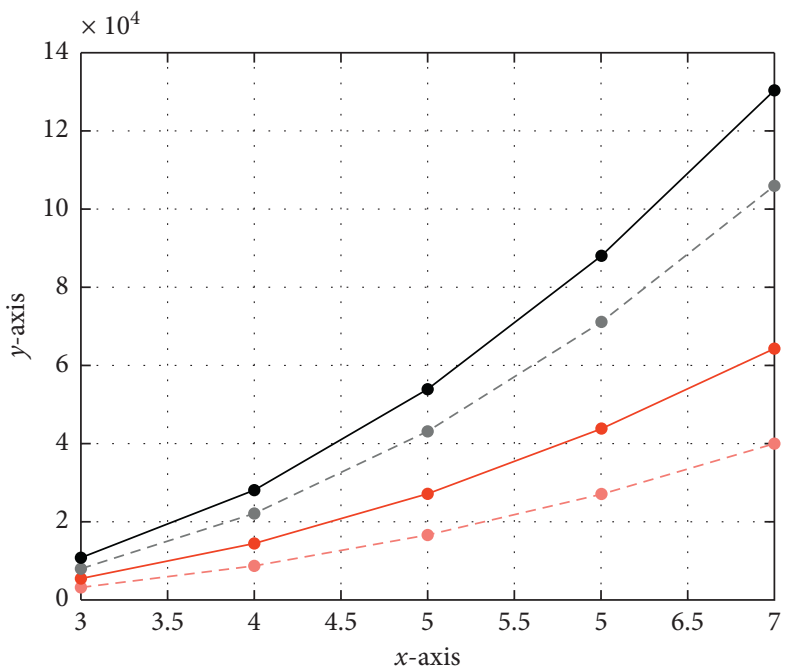

(b)

FIGURE 9: Compression between $F$-index of $F_{1}$-sum and $F_{2}$-sum graphs. (a) $F\left(P_{n_{1_{1}}} \otimes P_{n_{2}}\right.$ ) and $F\left(P_{n_{1_{2}}} \otimes P_{n_{2}}\right)$ are denoted by light green dotted line and dark green solid line, and $F\left(P_{n_{R_{1}}} \otimes P_{n_{2}}\right)$ and $F\left(P_{n_{R_{2}}} \otimes P_{n_{2}}\right)$ are denoted by sky blue dotted line and blue solid line, respectively. (b) $F\left(P_{n_{1_{1}}} \otimes P_{n_{2}}\right)$ and $F\left(P_{n_{1_{Q_{2}}}} \otimes P_{n_{2}}\right)$ are denoted by pink dotted line and red solid line, and $F\left(P_{n_{1_{1}}} \otimes P_{n_{2}}\right)$ and $F\left(P_{n_{1_{2}}} \otimes P_{n_{2}}\right)$ by grey dotted line and black solid line, respectively.

\section{Applications and Discussion}

In this paper, we computed the F-index of the generalized sum graphs. Assume that $H_{1}=P_{n_{1}}$ and $H_{2}=P_{n_{2}}$ are particular alkane called as paths of orders $n_{1}, n_{2} \geq 3$, and $k \geq 1$, respectively. Then, the following outcomes are the direct consequences of the achieved results:

(i)) $F\left(P_{n_{1}} \otimes P_{n_{2}}\right)=128 k\left(n_{1}-1\right)+250\left(n_{2}+n_{1}\right)+$ $512\left(n_{2}-2\right)\left(n_{1}-2\right)+216 k\left(n_{1}-1\right)\left(n_{2}-2\right)-892$ (ii) $F\left(P_{n_{1_{R}}} \otimes P_{n_{2}}\right)=128 k\left(n_{1}-1\right)+216 k\left(n_{1}-1\right)\left(n_{2}-\right.$ 2) $+2744\left(n_{2}-2\right)\left(n_{1}-2\right)+1458 n_{1}+1024 n_{2}-4464$

(iii) $F\left(P_{n_{1}} \otimes P_{n_{2}}\right)=432 k\left(n_{1}-1\right)+729 k\left(n_{1}-1\right)\left(n_{2}-\right.$ 2) $+512\left(n_{2}-2\right)\left(n_{1}-2\right)+250\left(n_{1}+n_{2}\right)-892$

(iv) $F\left(P_{n_{1}} \otimes P_{n_{2}}\right)=432 k\left(n_{1}-1\right)+729 k\left(n_{1}-1\right)\left(n_{2}-\right.$ $2)+2744\left(n_{2}-2\right)\left(n_{1}-2\right)+1458 n_{1}+1024 n_{2}-4464$

(1) Four subdivision operations $\left(S_{1}(G), R_{1}(G)\right.$, $\left.Q_{1}(G), T_{1}(G)\right)$ are restricted in case of newly 
addition of vertices upto $k=1$, while four subdivision operations $\left(S_{k}(G), R_{k}(G), Q_{k}(G), T_{k}(G)\right)$ are not restricted in case of newly addition of vertices as $k$ can be any natural number.

(2) For a certain graph $G$, newly constructed graphs $\left(S_{1}(G), R_{1}(G), Q_{1}(G), T_{1}(G)\right)$ will have specific number of edges. While, in $\left(S_{k}(G), R_{k}(G)\right.$, $\left.Q_{k}(G), T_{k}(G)\right)$, the number of edges will be varying according to $k$.

(3) Molecular structure of $\left(S_{k}(G), R_{k}(G), Q_{k}(G)\right.$, $\left.T_{k}(G)\right)$ is more general as compared to the $\left(S_{1}(G)\right.$, $\left.R_{1}(G), Q_{1}(G), T_{1}(G)\right)$.

(4) Molecular structure of $F_{k}$-sum is more complex and general as compared to the molecular structure of $F_{1}$-sum.

(5) In this paper, main results $F\left(H_{1_{s k}} \otimes H_{2}\right)$, $F\left(H_{1_{Q k}} \otimes H_{2}\right), \quad F\left(H_{1_{R k}} \otimes H_{2}\right), \quad$ and $F\left(H_{1_{T k}} \otimes H_{2}\right)$ based on strong product are generalization of $F\left(H_{1_{S 1}} \otimes H_{2}\right), \quad F\left(H_{1_{Q 1}} \otimes H_{2}\right), \quad F\left(H_{1_{R 1}} \otimes H_{2}\right), \quad$ and $F\left(H_{1_{T 1}} \otimes H_{2}\right)$ based on strong product.

Now, we present its tabular form, Table 1 and Table 2 and graphical representations in Figures 8 and 9 for $k=1$ and $k=2$.

In this paper, we computed the F-index of the subdivision-related generalized $F$-sum graphs based on strong product. However, the problem is still open for other topological indices on the generalized $F$-sum graphs.

\section{Data Availability}

The data used to support the findings of this study are included within the article. However, additional data may be provided by the corresponding author upon request.

\section{Conflicts of Interest}

The authors declare that they have no conflicts of interest.

\section{References}

[1] N. Trinajstic, Chemical Graph Theory, 2nd edition, 1992.

[2] I. Gutman, B. Ruščić, N. Trinajstić, and C. F. Wilcox, "Graph theory and molecular orbitals. XII. Acyclic polyenes," The Journal of Chemical Physics, vol. 62, no. 9, pp. 3399-3405, 1975.

[3] R. Todeschini and V. Consonni, Molecular Descriptors for Chemoinformatics, Wiley-VCH Verlag $\mathrm{GmbH}$, Weinheim, Germany, 2nd edition, 2009.

[4] I. Gutman, "Degree-based topological indices," Croatica Chemica Acta, vol. 86, no. 4, pp. 351-361, 2013.

[5] H. Wiener, "Structural determination of paraffin boiling points," Journal of the American Chemical Society, vol. 69, no. $1,1947$.

[6] I. Gutman and N. Trinajstić, "Graph theory and molecular orbitals,” New Concepts, vol. 2, pp. 49-93, 1973.

[7] W. Yan, B.-Y. Yang, and Y.-N. Yeh, "The behavior of Wiener indices and polynomials of graphs under five graph decorations," Applied Mathematics Letters, vol. 20, no. 3, pp. 290-295, 2007.
[8] M. Eliasi and B. Taeri, "Four new sums of graphs and their Wiener indices," Discrete Applied Mathematics, vol. 157, no. 4, pp. 794-803, 2009.

[9] H. Deng, D. Sarala, S. K. Ayyaswamy, and S. Balachandran, "The Zagreb indices of four operations on graphs," Applied Mathematics and Computation, vol. 275, pp. 422-431, 2016.

[10] S. Akhter and M. Imran, "Computing the forgotten topological index of four operations on graphs," AKCE International Journal of Graphs and Combinatorics, vol. 14, no. 1, pp. 70-79, 2017.

[11] J.-B. Liu, S. Javed, M. Javaid, and K. Shabbir, "Computing first general Zagreb index of operations on graphs," IEEE Access, vol. 7, pp. 47494-47502, 2019.

[12] Y.-M. Chu, S. Javed, M. Javaid, and M. Kamran Siddiqui, "On bounds for topological descriptors of $\varphi$-sum graphs," Journal of Taibah University for Science, vol. 14, no. 1, pp. 1288-1301, 2020.

[13] D. Sarala, H. Deng, C. Natarajan, and S. K. Ayyaswamy, " $F$ index of graphs based on four new operations related to the strong product," AKCE International Journal of Graphs and Combinatorics, vol. 17, no. 1, pp. 25-37, 2020.

[14] M. Munir, W. Nazeer, S. Rafique, and S. Kang, "M-polynomial and degree-based topological indices of polyhex nanotubes," Symmetry, vol. 8, no. 12, p. 149, 2016.

[15] YC. Kwun, M. Munir, W. Nazeer, S. Rafique, and S. M. Kang, "M-Polynomials and topological indices of V-phenylenic nanotubes and nanotori," Scientific Reports, vol. 7, no. 1, pp. 1-9, 2017.

[16] H. M. Awais, M. Javaid, and A. Akbar, "First general Zagreb index of generalized F-sum graphs," Discrete Dynamics in Nature and Society, vol. 2020, Article ID 2954975, 16 pages, 2020.

[17] J. H. Tang, U. Ali, M. Javaid, and K. Shabbir, "Zagreb connection indices of subdivision and semi-total point operations on graphs," Journal of Chemistry, vol. 2019, Article ID 9846913, 14 pages, 2019.

[18] M. F. Nadeem, S. Zafar, and Z. Zahid, "On the edge version of geometric-arithmetic index of nanocones," Studia Universitatis Babes-Bolyai, Chemia, vol. 61, no. 1, pp. 273-282, 2016.

[19] J. B. Liu, S. Akram, M. Javaid, A. Raheem, and R. Hasni, "Bounds of degree-based molecular descriptors for generalized-sum graphs," Discrete Dynamics in Nature and Society, vol. 2021, Article ID 8821020, 17 pages, 2021.

[20] A. Ahmad, K. Elahi, R. Hasni, and M. F. Nadeem, "Computing the degree based topological indices of line graph of benzene ring embedded in P-type-surface in 2D network," Journal of Information and Optimization Sciences, vol. 40, no. 7, pp. 1511-1528, 2019.

[21] J. B. Liu, M. Javaid, and H. M. Awais, "Computing Zagreb indices of the subdivision-related generalized operations of graphs,” IEEE Access, vol. 7, pp. 105479-105488, 2019.

[22] H. M. Awais, M. Javaid, and M. Jamal, "Forgotten index of generalized F-sum graphs," Journal of Prime Research in Mathematics, vol. 15, pp. 115-128, 2019.

[23] H. M. Awais, M. Javaid, and A. Raheem, "Hyper-Zagreb index of graphs based on generalized subdivision related operations," Punjab University Journal of Mathematics, vol. 52, no. 5, pp. 89-103, 2019.

[24] X. Zhang, H. M. Awais, M. Javaid, and M. K. Siddiqui, "Multiplicative Zagreb indices of molecular graphs," Journal of Chemistry, vol. 2019, Article ID 5294198, 19 pages, 2019.

[25] M. Javaid, S. Javed, A. M. Alanazi, and M. R. Alotaibi, "Computing analysis of Zagreb indices for generalized sum 
graphs under strong product," Journal of Chemistry, vol. 2021, Article ID 6663624, 20 pages, 2021.

[26] I. Gutman and N. Trinajstić, "Graph theory and molecular orbitals. Total $\varphi$-electron energy of alternant hydrocarbons," Chemical Physics Letters, vol. 17, no. 4, pp. 535-538, 1972.

[27] B. Furtula and I. Gutman, "A forgotten topological index," Journal of Mathematical Chemistry, vol. 53, no. 4, pp. 11841190, 2015. 